\title{
Towards Real-World Applications of Online Learning Spiral Recurrent Neural Networks
}

\author{
Rudolf SOLLACHER ${ }^{1}$, Huaien GAO ${ }^{2}$ \\ ${ }^{1}$ Siemens AG, Corporate Technology, Munich, Germany; ${ }^{2}$ Department of Computer Science, Ludwig-Maximilians University, Mu- \\ nich, Germany. \\ Email: Rudolf.Sollacher@siemens.com,gheken@hotmail.com
}

Received October 21 $1^{\text {st }}$ 2008; revised January 19 ${ }^{\text {th }}$, 2009; accepted January $21^{\text {st }}, 2009$.

\begin{abstract}
Distributed intelligent systems like self-organizing wireless sensor and actuator networks are supposed to work mostly autonomous even under changing environmental conditions. This requires robust and efficient self-learning capabilities implementable on embedded systems with limited memory and computational power. We present a new solution called Spiral Recurrent Neural Networks (SpiralRNN) with an online learning based on an extended Kalman filter and gradients as in Real Time Recurrent Learning. We illustrate its stability and performance using artificial and real-life time series and compare its prediction performance to other approaches. SpiralRNNs perform very stable and show an accuracy which is superior or similar to other state-of-the-art approaches. In a memory capacity evaluation the number of simultaneously memorized and accurately retrievable trajectories of fixed length was counted. This capacity turned out to be a linear function of the size of the recurrent hidden layer, with a memory-to-size ratio of 0.64 for shorter trajectories and 0.31 for longer trajectories. Finally, we describe two potential applications in building automation and logistics and report on an implementation of online learning SpiralRNN on a wireless sensor platform under the TinyOS embedded operating system.
\end{abstract}

Keywords: recurrent neural network, online learning, prediction, sensor actuator network

\section{Introduction}

Visions like the "intelligent factory" [1] or "ambient intelligence" [2] rely heavily on embedded systems with a high degree of autonomy. Of particular interest are energy autarkic self-organizing wireless sensor networks due to their simplified installation and robustness during operation. These consist of so called "sensor nodes", hardware platforms with usually a battery, a microprocessor, a transceiver module and one or more sensors. They are able to communicate wirelessly with each other; suitable protocols enable them to set up a network by themselves. If necessary, data are relayed by other nodes to a destination. Data may also be processed locally or inside the network.

A very useful kind of data processing is forecasting data. Examples for applications in building automation and logistics are given at the end of this paper. Solutions for this task have to fulfill at least the following requirements in order to be really useful:

R1 The limited computational power and memory of these sensor nodes puts a limit on the computational complexity of the forecasting solution.
R2 As the environment of the sensor network is usually unknown in advance the solution has to learn a forecast model online and in an efficient way.

Neural networks, and in particular recurrent neural networks, have proven their suitability at least for offline learning forecast tasks. Examples can be found in [3] or [4]. However, traditional approaches like Elman or standard recurrent neural networks (SRN) [5], time delay neural networks (TDNN) [6], block-diagonal recurrent neural networks (BDRNN) [7] or echo state neural networks (ESN) [8] have deficiencies with respect to the requirements above. For a TDNN one has to specify the length of the history to be considered as input. If this is chosen too small, some time series like spike time series with large inter-spike intervals cannot be predicted reliably. Elman networks avoid this problem due to their recurrent hidden layer; this allows them to adjust their short term memory in a data driven way by online learning. However, their basically unbounded recurrent weight matrix sometimes leads to dynamically unstable behavior making them less attractive for online learning 
purposes. Echo state networks try to cure this deficiency by choosing random but constant weights for the recurrent weight matrix, but such that its absolute eigenvalues are smaller than one; only the weights of the output layer are trained. Unfortunately, with this approach the recurrent layer has to be large enough in order to provide a rich reservoir of responses from which to construct the output signals. Block diagonal recurrent neural networks try to cure the dynamical instabilities by a specific structure of the recurrent layer. The corresponding weight matrix is zero except for $2 \times 2$ diagonal block matrices. These blocks can be trained but there is a constraint on their maximum eigenvalue. This constraint is either implemented implicitly via training or explicitly via a suitable parametrization of the weights. For online learning the scaled orthogonal version described in [7] is more useful. However, this structure seemed to be too constrained leading to poor performance for some prediction problems. This motivated us to develop a new approach called Spiral Recurrent Neural Network (SpiralRNN) [9].

\section{Spiral Recurrent Neural Networks (SpiralRNN)}

\subsection{Architecture}

Like other Recurrent Neural Network (RNN) models, SpiralRNN consists of an input layer feeding into a recurrent hidden layer and an output layer which receives input from the recurrent hidden layer. The hidden neuron states $\mathbf{s}_{t}$ and the output neuron states $\mathbf{x}_{t}$ satisfy the following update equations:

$$
\begin{aligned}
& \mathbf{s}_{t}=g\left(W_{\text {hid }} \mathbf{s}_{t-1}+W_{\text {in }} \mathbf{x}_{t-1}+\mathbf{b}_{\text {hid }}\right) \\
& \mathbf{x}_{t}=h\left(W_{\text {out }} \mathbf{s}_{t}+\mathbf{b}_{\text {out }}\right)
\end{aligned}
$$

Here, $\mathbf{b}_{\text {hid }}$ and $\mathbf{b}_{\text {out }}$ are bias vectors, matrices $W_{\text {in }}$, $W_{\text {hid }}$ and $W_{\text {out }}$ represent synaptic connections between layers and $g()$ and $h()$ are activation functions. Using the data $\hat{\mathbf{x}}_{t-1}$ instead of the previous network output $\mathbf{x}_{t-1}$ as input in Equation (1) corresponds to teacher forcing; this is used whenever data are available.

The main difference to other approaches lies in the structure of the recurrent hidden matrix $W_{\text {hid }}$. Motivated by the analysis underlying the development of echo state neural networks (ESN) [8], we have chosen a constraint on the weights of the recurrent layer in order to guarantee stability, respectively the "echo state" property, while still allowing the weights to be trained. With the following structure,

$$
W_{\text {hid }}=\left(\begin{array}{cccc}
0 & \beta_{l-1} & \ldots & \beta_{1} \\
\beta_{1} & 0 & \ddots & \vdots \\
\vdots & \ddots & \ddots & \beta_{l-1} \\
\beta_{l-1} & \ldots & \beta_{1} & 0
\end{array}\right)_{l \times l},
$$

with $l$ the number of hidden nodes in the recurrent layer, $\beta_{k}=\gamma \tanh \left(\xi_{k}\right), k \in[1, l-1], \quad \xi_{k}$ trainable parameters and a suitable fixed $\gamma \in \mathbf{R}^{+}$, one can guarantee a bounded eigenvalue spectrum of $W_{\text {hid }}$ and thus stability in the sense of the "echo state" property:

$$
\left|\lambda^{\vec{\beta}}\right| \leq \sum_{k=1}^{l-1}\left|\beta_{k}\right|=\gamma \sum_{k=1}^{l-1} \tanh \left(\left|\xi_{k}\right|\right) \leq \gamma(l-1)
$$

The structure of $W_{\text {hid }}$ is best illustrated with Figure 1, also giving an idea for the choice of the name "SpiralRNN".

It turned out that a block structure in case of several inputs is a good compromise concerning computational complexity and performance (see Figure 2). Although the recurrent hidden layer has this block structure, all input neurons are coupled to all hidden neurons; the same holds for the output neurons.

\subsection{Online Learning}

Due to the second requirement mentioned in the introduction the learning of the neural network weights has to be online. We understand online learning as a continuous update of weights controlled only by the data. Realtime Recurrent Learning (RTRL) is based on an approximate error gradient taking into account all previous steps; it sometimes shows slow or poor convergence. Particle filtering and evolutionary approaches require much memory

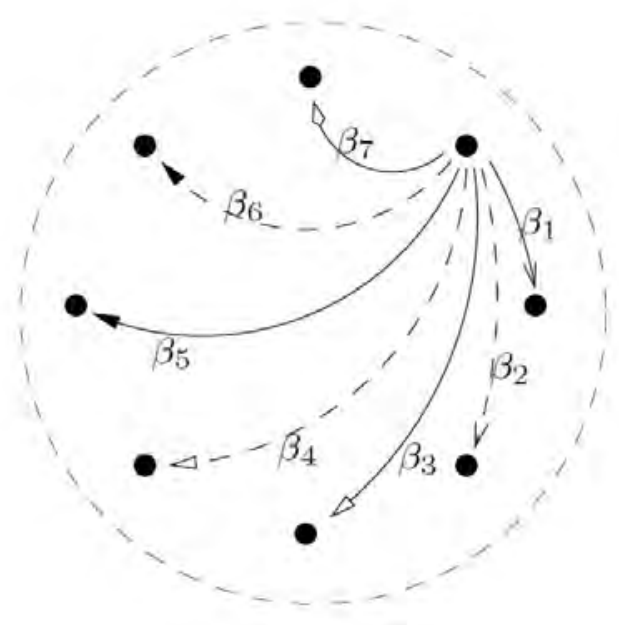

Figure 1. Structure of SpiralRNN recurrent hidden layer for 1-dimensional input; only the connections rooted in one neuron are shown 


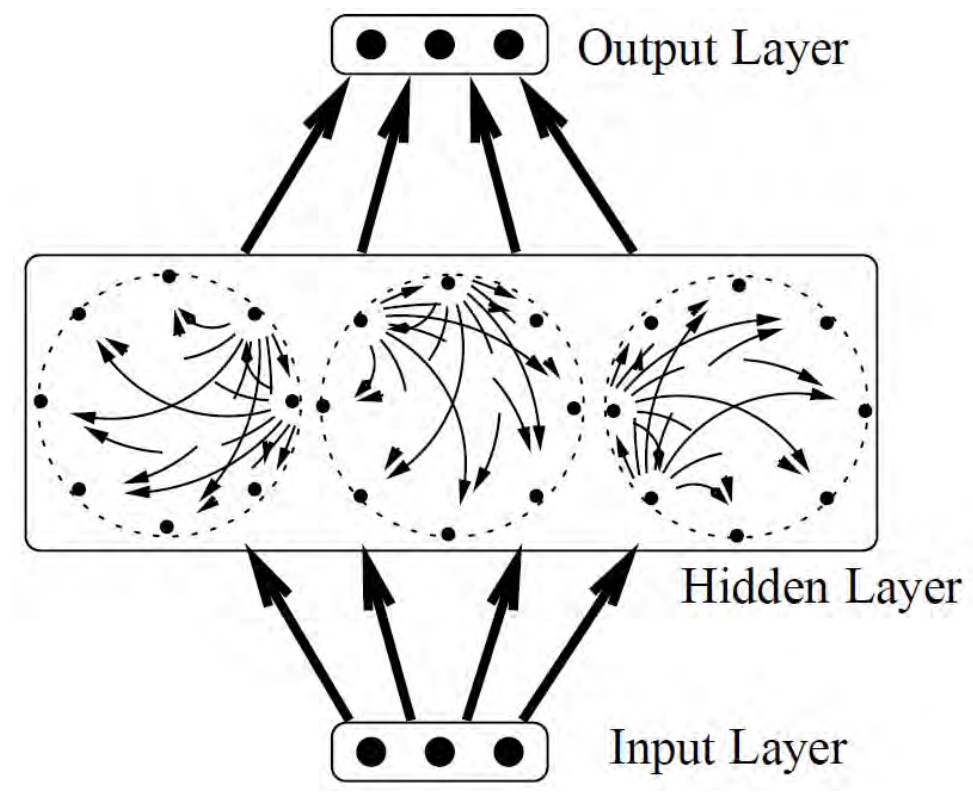

Figure 2. SpiralRNN for 3-dimensional input; the recurrent hidden matrix is split into 3 blocks but all other layers are fully connected

in order to provide reasonable behavior. As explained in [9] the optimal choice is an extended Kalman filter $(E K F)$ based on gradients calculated like in Realtime Recurrent Learning (s. appendix).

According to this derivation, the iterative update rule for the weight vector $\mathbf{w}_{t}$, representing all weight matrices $W_{\text {in }}, W_{\text {hid }}$ and $W_{\text {out }}$ and all bias vectors $b_{\text {hid }}$ and $b_{\text {out }}$, is the following:

$$
\begin{aligned}
P_{t}^{\dagger} & =P_{t-1}+Q_{t} \\
P_{t} & =P_{t}^{\dagger}-P_{t}^{\dagger} \Psi^{T}\left(\Psi P_{t}^{\dagger} \Psi^{T}+R_{t}\right)^{-1} \Psi P_{t} \\
\mathrm{w}_{t} & =\mathrm{w}_{t-1}+P_{t} \Psi^{T} R_{t}^{-1}\left(\hat{x}_{t}-\mathrm{x}_{t}\right)
\end{aligned}
$$

$\mathbf{x}_{t}$ is the output vector of the Spiral RNN and $\hat{\mathbf{x}}_{t}$ are the data. $P_{t}$ is the covariance matrix of $\mathbf{w}_{t}$ representing its uncertainty and $Q_{t}$ is the incremental increase in this uncertainty. $R_{t}$ is the measurement error covariance matrix. $\Psi$ represents the gradient of the output $\mathbf{x}_{t}$ w.r.t. $\quad \mathbf{w}_{t-1}$ (notice the indexing):

$$
\begin{gathered}
\Psi=\frac{d \mathbf{x}_{t}}{d \mathbf{w}_{t-1}}=\frac{\partial \mathbf{x}_{t}}{\partial \mathbf{s}_{t}} \frac{d \mathbf{s}_{t}}{d \mathbf{w}_{t-1}}+\frac{\partial \mathbf{x}_{t}}{\partial \mathbf{w}_{t-1}} \\
\frac{d s_{t}}{d w_{t-1}}=\frac{\partial s_{t}}{\partial w_{t-1}}+\frac{\partial s_{t}}{\partial s_{t-1}} \frac{d s_{t-1}}{d w_{t-1}} \\
\approx \frac{\partial s_{t}}{\partial w_{t-1}}+\frac{\partial s_{t}}{\partial s_{t-1}} \frac{d s_{t-1}}{d w_{t-2}}
\end{gathered}
$$

The last line in Equation (6) is the gradient calculated in real time recurrent learning (RTRL) [10]. This approximation is justified if the weight vector is changing slowly which holds at least for the later phase of the converging learning process.

It is worth mentioning that these iterative Kalman filter equations for the update of expected weight values and their covariance matrix are derived analytically under three reasonable approximations: 1) the distribution of noise on the data is Gaussian, 2) the weights change slowly and 3) the distribution of weights representing their uncertainty is unimodal and also Gaussian. While 1) is a standard assumption, the other two approximations deserve some explanation: 2) is true if the process generating the data is stationary and if the model is powerful enough to reproduce the data; only in the initial phase of learning and for very non-stationary processes this assumption might be questionable. Usually, 3) is only true for a local neighborhood around the true weight vector; in general, the distribution will be multi-modal implying several competing weight vectors and thus models. However, for complexity reasons we have to focus on one set of weights; we have no guarantee that we get the optimal model but we will find a "locally" optimal model in the sense, that small changes of the weights lead to a decrease in performance. As a consequence we will end up with a distribution of different models in repeated experiments with different initial conditions or noise. The reader should notice, that there will be always several degenerate solutions, because a renumbering of 
the hidden neurons yields exactly the same dynamical model but the weight vector is changed; the components have just been reshuffled. This is actually an advantage for learning because it is sufficient to reach one of these degenerate solutions.

\subsection{Performance}

Spiral RNN have been compared to other architectures like ESN, Elman networks and BDRNN [9]. TDNN is not included due to the need to fix the number of historic inputs in advance. The generalization ability of these networks was tested with three artificial time series: A spike time series with spike intervals of 21 time steps (20 zeros followed by a one), and the chaotic Mackey-Glass and Lorenz time series. The one-dimensional MackeyGlass time series is given by

$$
\dot{x}(t)=\frac{a x(t-\tau)}{1+x(t-\tau)^{c}}-b x(t)
$$

with the parameters $a=0.2, b=0.1, c=10$ and $\tau=17$. The differential equation was solved numerically using Euler's method with step size 1 . All time series values before start time were set to 0.5 . The time series was normalized by its standard deviation.

The three-dimensional Lorenz time series is defined by the following equations:

$$
\begin{gathered}
\dot{x}=s(y-x) \\
\dot{y}=(r-z) x-y \\
\dot{z}=x y-b z
\end{gathered}
$$

The parameters were set to $s=16, r=40, b=6$ and the initial values were $x_{0}=y_{0}=0.1$ and $z_{0}=-0.1$. Integration was done using Euler's method with step size 0.01 . The data were finally scaled down by factor of 20 .

All time series are corrupted - after normalization - by normally distributed noise with a standard deviation of 0.01 .

As the computational complexity depends mainly on the online learning step we compared different networks with roughly the same number of trainable parameters. For the activation function in the hidden layer we took $\tanh ()$ and in the output layer we took the identity map. The blocks in the diagonal of the recurrent weight matrix for the BDRNN were parameterized as

$$
\left(\begin{array}{cc}
\tanh (\Phi) \sin (\Theta) & \tanh (\Phi) \cos (\Theta) \\
-\tanh (\Phi) \cos (\Theta) & \tanh (\Phi) \sin (\Theta)
\end{array}\right)
$$

with a pair of trainable parameters $\Phi$ and $\Theta$ independently for each block.

For the ESN model, the recurrent weight matrix was initialized with random numbers uniformly distributed in the interval $[-1,1]$ with a sparsity degree of $95 \%$; the matrix was rescaled such that the maximum absolute eigenvalue was 0.8 . All other weights are initialized with random numbers uniformly distributed in $[-0.1,0.1]$.

For SpiralRNNs, a value $\gamma=\frac{1}{l-1}$ guarantees that the maximum eigenvalue of the recurrent weight matrix is smaller than one. However, the actual eigenvalues can be much smaller than one. Experiments have shown good and reliable performance with $\gamma=1$.

The online learning method was the same as described above for all networks with appropriate gradients depending on the architecture. For the Kalman filter, the covariance matrix $P_{t}$ was initialized with the identity matrix Id and the model noise covariance matrix $Q_{t}=10^{-8} I d$ was chosen to be constant. The measurement covariance matrix $R_{t}$ was initialized to $10^{-2} I d$ and updated according to

$$
R_{t}=(1-\alpha) R_{t-1}+\alpha \mathrm{e} \times \mathrm{e}^{T},
$$

with $\mathrm{e}=\mathrm{x}_{t}-\hat{\mathrm{x}}_{t}$ the forecast error at time $t$ and $\alpha=0.01$.

During online learning we used teacher forcing. After some specific time steps we performed multi-step forecast tests using the output as input for the next time step. After each test we returned to the beginning of the test and continued online learning with teacher forcing.

The prediction performance during the tests is measured with the logarithmic normalized mean square error (logNMSE):

$$
\varepsilon=\frac{1}{d} \sum_{i=1}^{d} \frac{1}{\Delta t} \sum_{t=t_{0}+1}^{t_{0}+\Delta t} \log _{10}\left(x_{i, t}-\hat{x}_{i, t}\right)^{2} / \sigma^{2}
$$

Here, $d$ is the dimension of the data, $t_{0}$ is the time before the test phase starts, $\Delta t$ is the maximum number of test steps and $\sigma$ is the standard deviation of the whole data set. This definition of an error measure takes into account that the prediction error in the test phases increases with time; as we do not want the error measure to be dominated by the large errors of the future prediction steps we have chosen a measure corresponding to a geometric mean instead of a traditional algebraic mean.

For the spike time series we performed tests with a duration of $\Delta t=2000$ time steps. For this time series many forecast models tend to predict a small constant value close to $1 / 21$. This can give a small error as this 
value is close to zero for 20 out of 21 time steps. Therefore, we evaluated the error measure for this particular time series only with the time steps when the spikes occurred; this provides a much more informative measure.

Figure 3 shows the results for networks with about 100 trainable weights. Obviously, SRN and SpiralRNN can predict the spike time series at least after $10^{4}$ time steps (recall that this corresponds to about 500 spike periods). On the other hand, ESN and BDRNN fail to predict the spike trains properly. Not quite unexpectedly, SRN, having more degrees of freedom, performs best. However, the results for SRN include only those runs which were successful; after longer training a significant fraction of runs failed due to dynamical instabilities. An encouraging observation is that SpiralRNN is pretty close in performance to the successful SRN runs, taking into account the logarithmic error measure.

Figure 4 shows the results for the Lorenz time series with tests consisting of 200 time steps. Here, SRN, BDRNN and SpiralRNN show similar behavior and good prediction capabilities at least after longer training. ESN outperforms the other approaches in the beginning of the training phase. This can be explained by the fact that the training of its output weights is basically a linear regression task and the Kalman filter learning is essentially the optimal method. However, ESN can hardly improve its performance even for long training periods. Obviously, for a good generalization it is necessary to adapt the recurrent hidden layer weights as well, a feature shared by the three other architectures. The intermediate increase of the error for all approaches can be explained with the characteristics of the time series which obviously explores new state space areas in these phases. Another interesting result is the test with the MackeyGlass time series. Figure 5 shows the result for networks with about 100 trainable weights and prediction tests for up to 200 time steps. Here, SpiralRNN clearly outperforms the other approaches, even SRN. For smaller network size with about 50 trainable weights, SRN is slightly better than SpiralRNN, while both approaches show almost as good behavior as SpiralRNN with about 100 weights; however, ESN and BDRNN perform similarly poor. An example of autonomous output of the SpiralRNN model on MackeyGlass time series is shown in Figure 6 where the difference between autonomous output and target data can be hardly identified until around time step 400 .

\subsection{Stability}

Stability of a trained SpiralRNN model has been demonstrated by autonomous tests with deviated initial input values. Recall that, in autonomous tests, the model uses the previous output value as the current input value. The only exception is the initial input value which is supplied from outside.

Figure 7 shows the autonomous outputs generated by a trained SpiralRNN model from the simulation with data set Spike21. Each sub-figure in Figure 7 shows the autonomous output result from simulation with different initial input values $\{ \pm 0.1, \pm 1, \pm 10, \pm 100\}$ respectively.

Without exception, all models with different initial inputs converge after an initial transition phase to the trained attractor regime with periodic spike output with period 21.

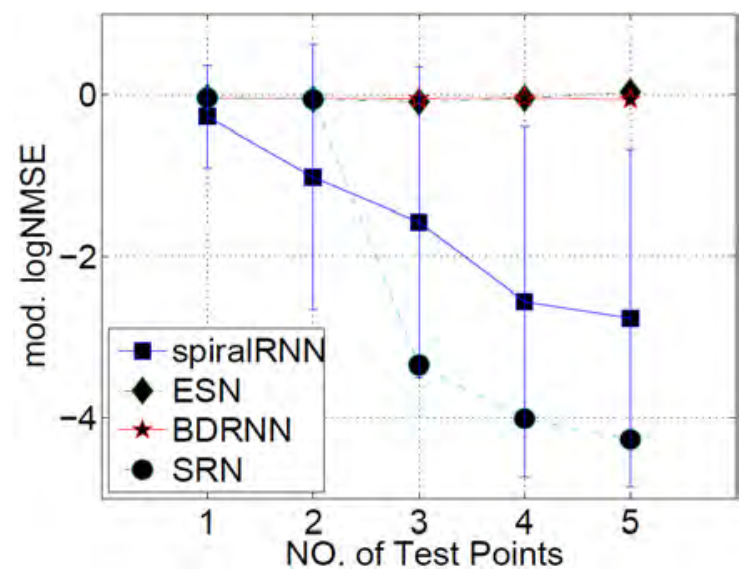

Figure 3. Modified logNMSE for the spike time series with period 21. The networks have about 100 trainable weights. The test points correspond to the times $\left\{10^{3}, 10^{3.5}, 10^{4}, 10^{4.5}, 10^{5}\right\}$

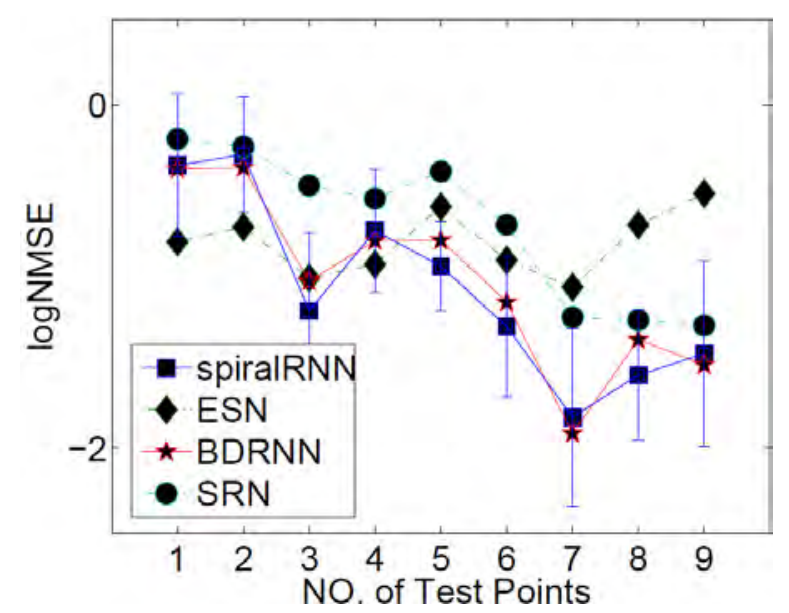

Figure 4. LogNMSE for the Lorenz time series and prediction tests with 200 time steps. The networks have about 100 trainable weights. The test points correspond to the times $\left\{10^{3}, \mathbf{1 0}^{3.25}, 10^{3.5}, \ldots, 10^{4.75}, 10^{5}\right\}$ 


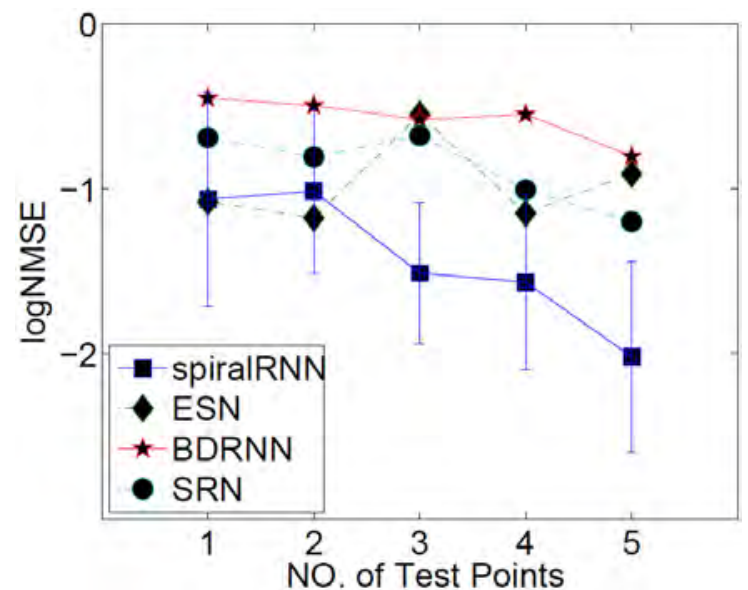

Figure 5. LogNMSE for the Mackey-Glass time series and prediction tests with 200 time steps. The networks have about 100 trainable weights. The test points correspond to the times $\left\{10^{3}, 10^{3.5}, 10^{4}, 10^{4.5}, 10^{5}\right\}$

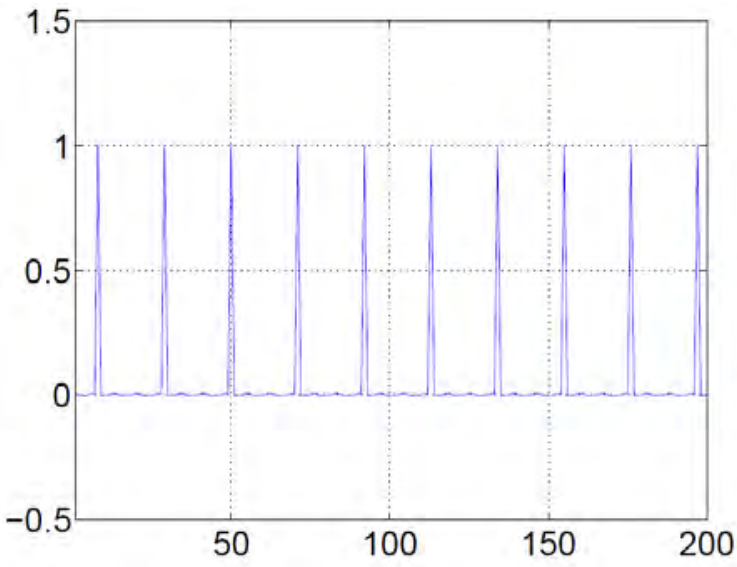

(a) -0.1

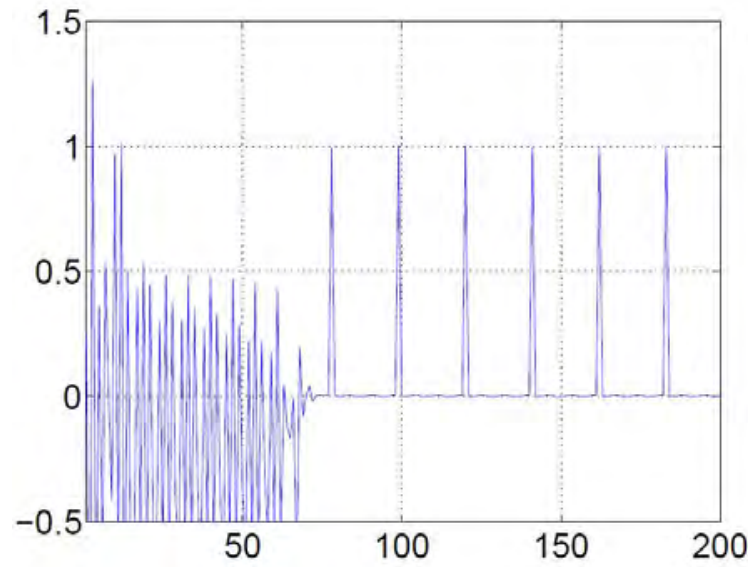

(c) -10

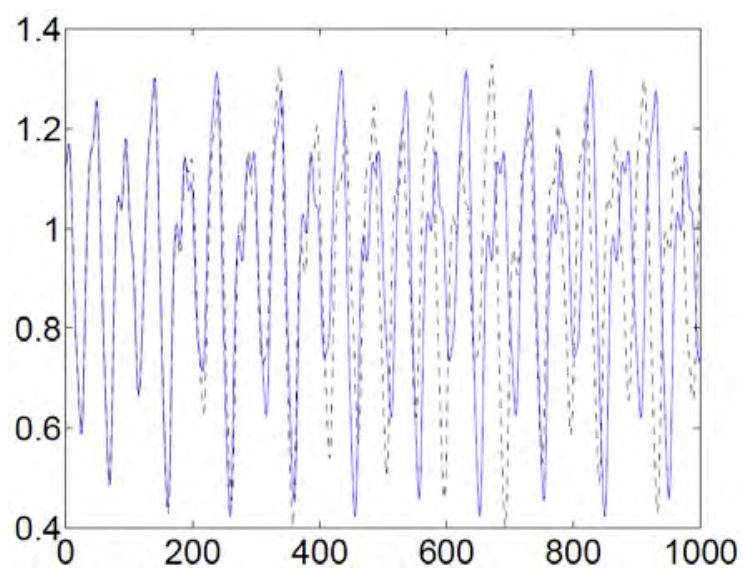

Figure 6. The autonomous output of a trained SpiralRNN model vs. corresponding target data. The solid line is autonomous output, and the dashed line is target data

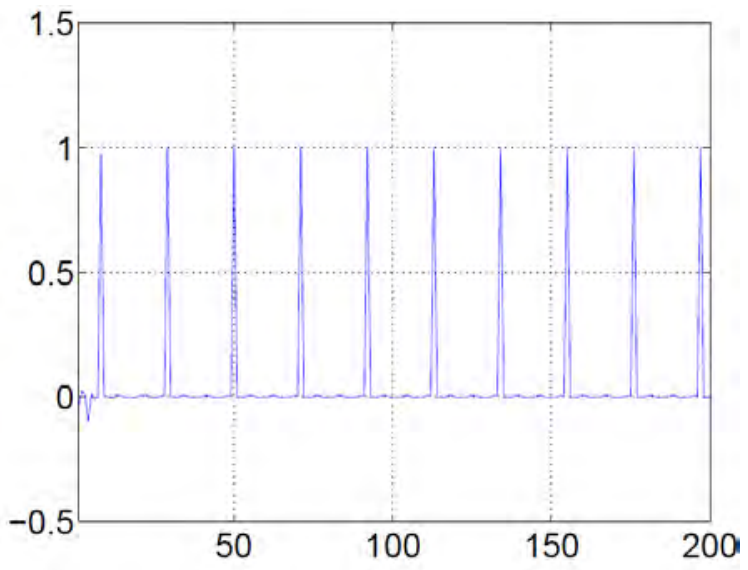

(b) -1

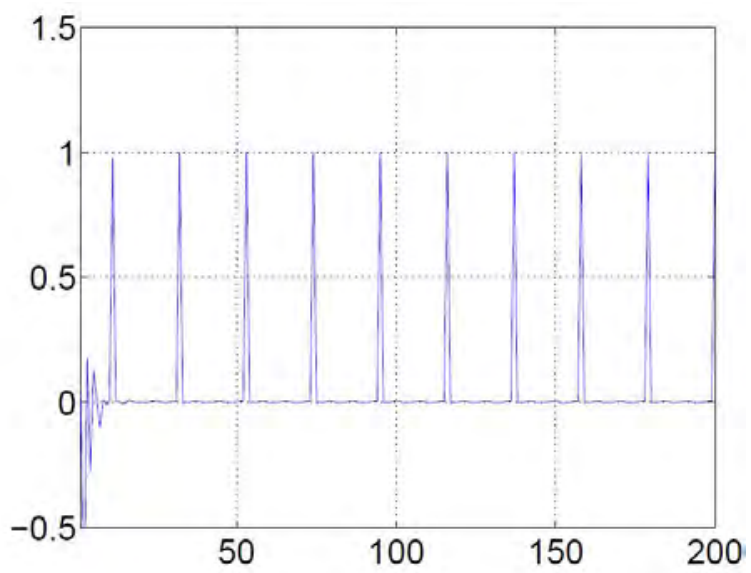

(d) $\mathbf{- 1 0 0}$ 


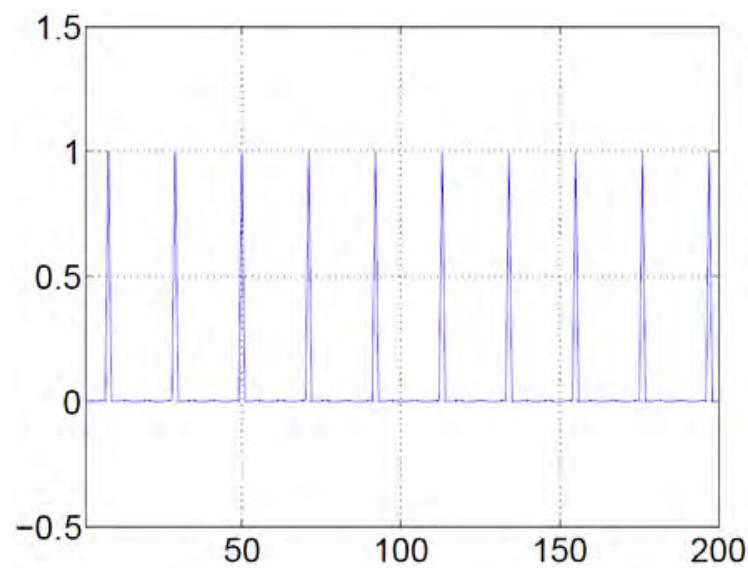

(e) 0.1

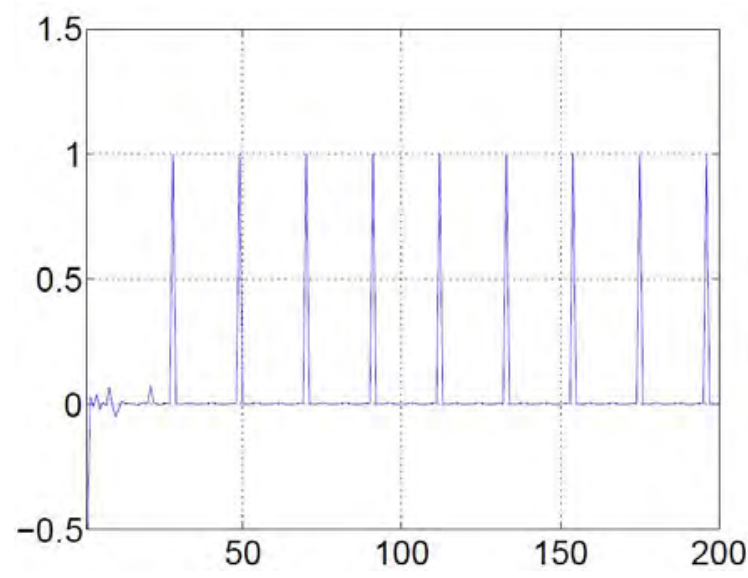

(g) 10

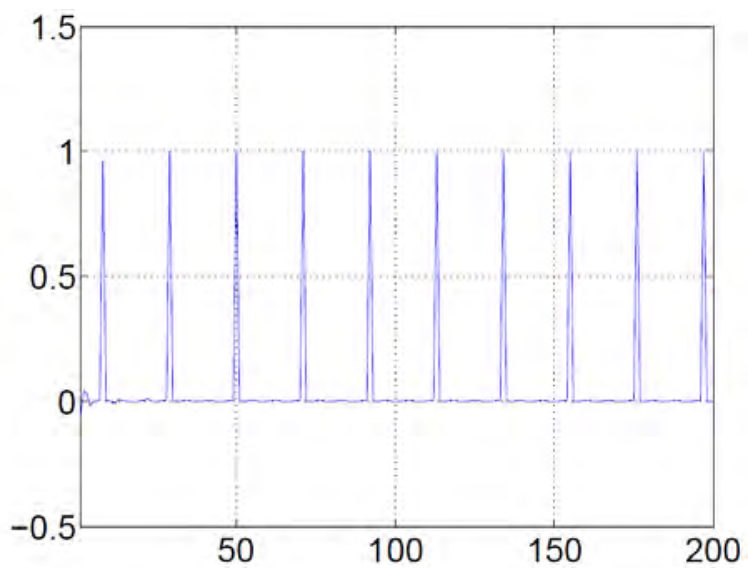

(f) 1

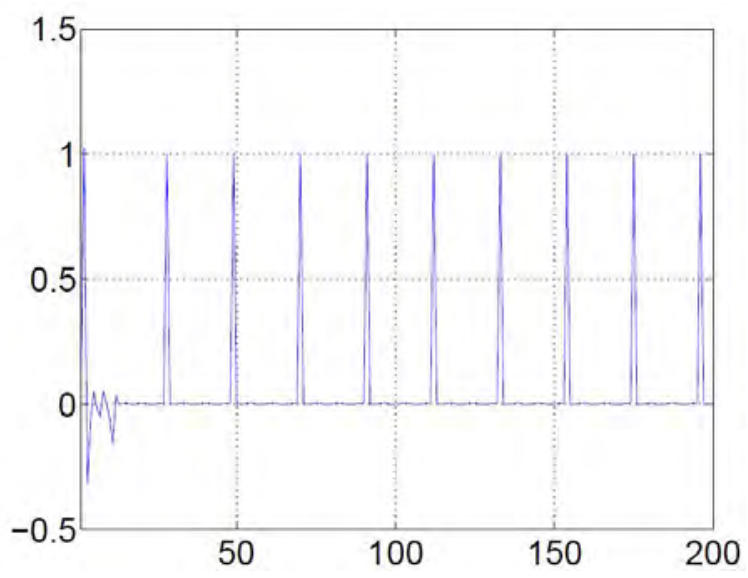

(h) 100

Figure 7. Stability test of a trained SpiralRNN model from the simulation with the Spike21 data set. The sub-figures show the results for different initial input values $\{-0.1,-1,-10,-100,+0.1,+1,+10,+100\}$ respectively. The $\mathrm{X}$-axis is the time in the autonomous tests, and the $\mathrm{Y}$-axis is the output

As another example, Figure 8 depicts the trajectories of two hidden-neuron activations during the autonomous test with a trained SpiralRNN model from a simulation with the Lorenz data set. In the test phase, the SpiralRNN model, is iterated autonomously for 1000 time steps starting with different initial input values. During the iteration, the recurrent layer neuron states are recorded. Sub-figures in Figure 8 show the trajectories of two neurons. The five initial time steps of the autonomous test are marked by black squares. Again, after some initial transition phase the trajectories always converge to an attractor corresponding to the Lorenz time series. Note that the sub-figures have different scale in order to display the initial transition path, and that the position of the attractor is in the range $[-0.2,0.6]$ for the $\mathrm{X}$-axis and $[-0.2,0.6]$ for the $\mathrm{Y}$-axis in all sub-figures.

\section{Application Examples}

\subsection{NN5 Competition}

NN5 competition ${ }^{1}$ puts emphasis on computational intelligence methods in data prediction. The data for this competition correspond to the amount of daily money withdrawal from ATM machines across England. These data exhibit strong periodical ${ }^{2}$ (ref. Figure 9) behavior:

\footnotetext{
${ }^{1}$ http://www.neural-forecasting-competition.com/

${ }^{2}$ Note that the Easter Fridays in 1996 to 1998 should have the indices "19", "376" and "753" in the given data and the Saturdays before
} Christmas day of 1996 and 1997 have the indices "283” and "648”. 
Strong weekly periodic behavior dominates the

$\mathrm{F}_{1} \quad$ frequency spectrum, usually with higher values on Thursday and/or Friday;

Important holidays such as the Christmas holi-

$\mathrm{F}_{2}$ days (including the New Year holiday) and the Easter holidays have a visible impact on the data;

Several of the time series such as time series

$\mathrm{F}_{3} \quad$ No. 93 and No. 89 show strong seasonal behavior, i.e. a yearly period;

Some of the time series (like No. 26 and No. 48)

$\mathrm{F}_{4}$ show a sudden change in their statistics, e.g. a shift in the mean value.

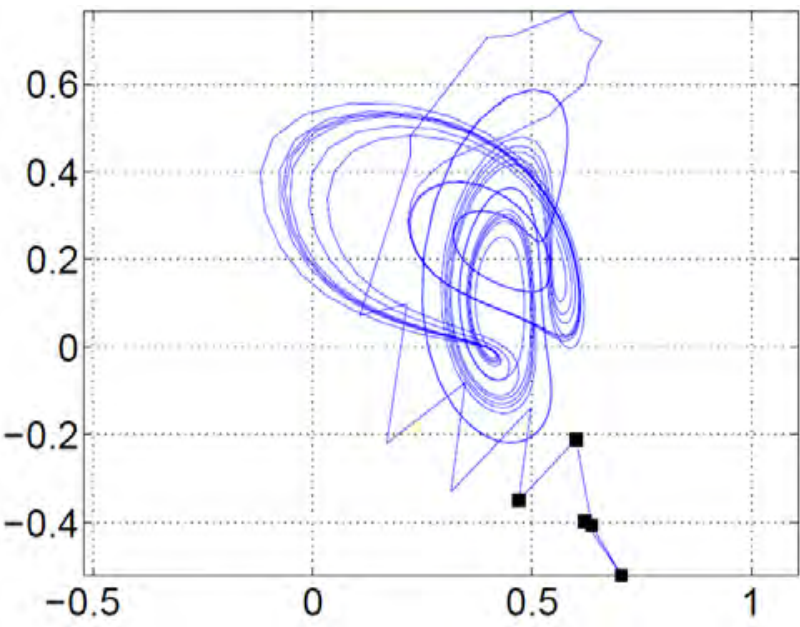

(a) $\{-4,-4,-4\}$

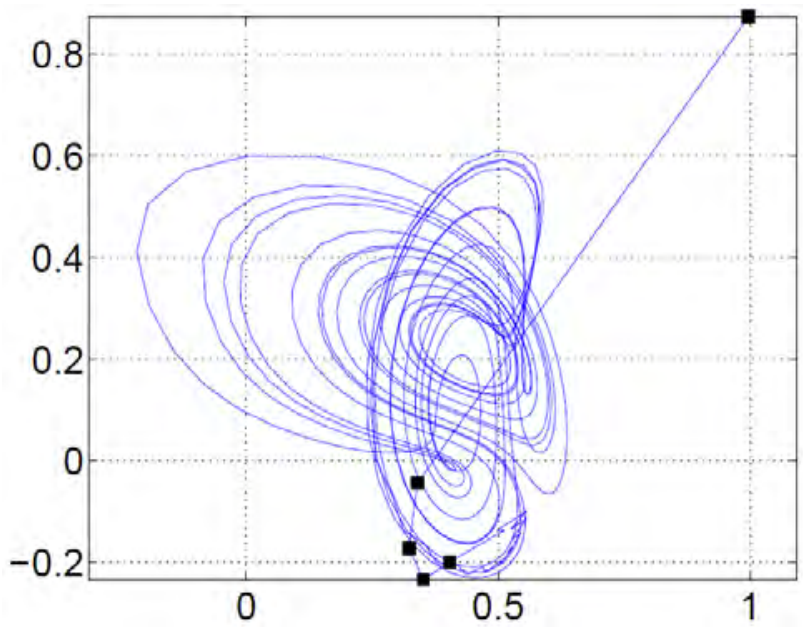

(c) $\{-4,+4,-4\}$
The dynamics associated with data have deterministic and stochastic components. In general, they will not be stationary, as for example more tourists are visiting this area or a new shopping mall has opened. There are in total 111 time series in the database, with each time series representing the withdrawal from one ATM machine and each data point in a particular time series indicating the withdrawal amount of the day from the particular ATM machine. All 111 time series were recorded from the 18th March 1996 until 22nd March 1998, and therefore contain 735 data points. The task of the competition is to predict the withdrawal of each ATM machine from 23rd March 1998 to 17th May 1998, 56 data points in total. The evaluation of prediction performance is based on the so-called SMAPE error value defined in Equation (14), where $x_{t}$ and $\hat{x}_{t}$ are respectively the predicted output and data.

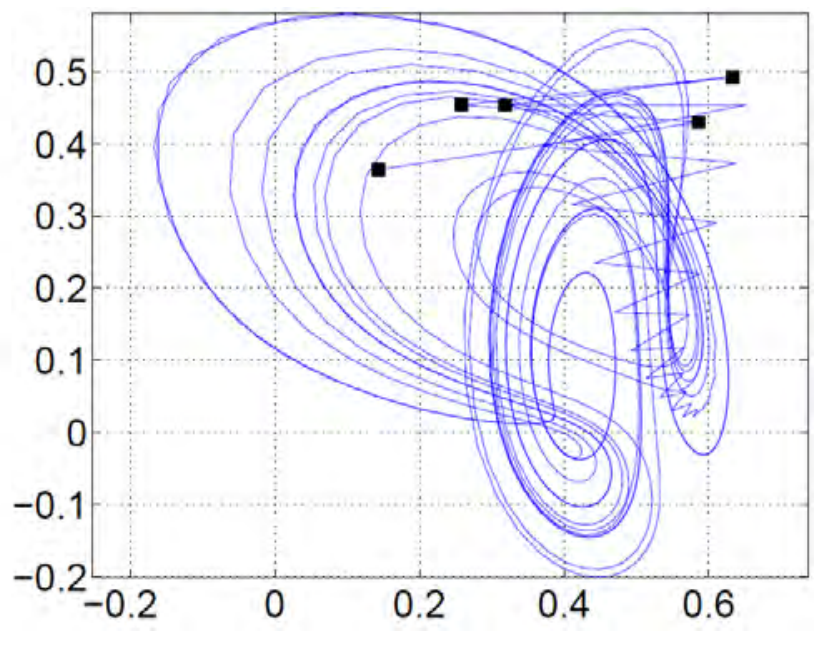

(b) $\{-4,-4,+4\}$

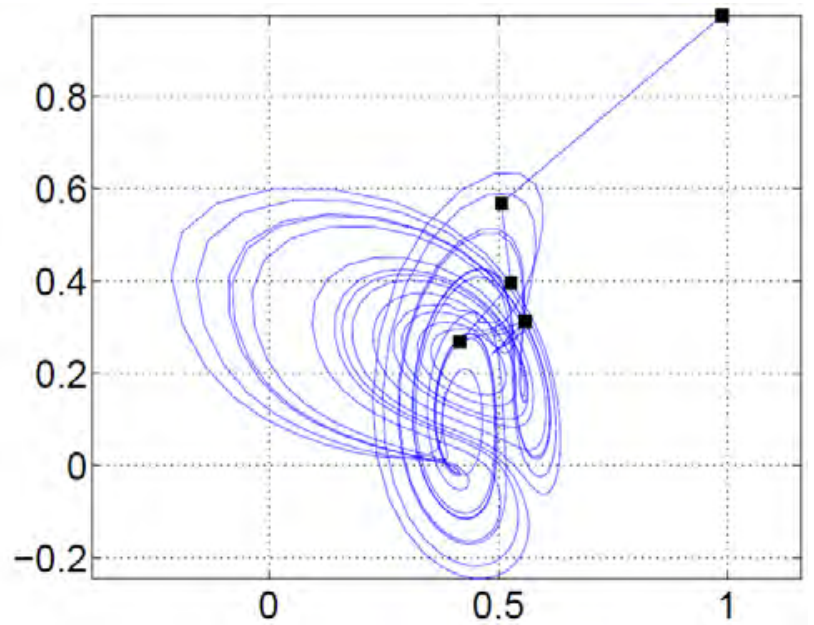

(d) $\{-4,+4,+4\}$ 


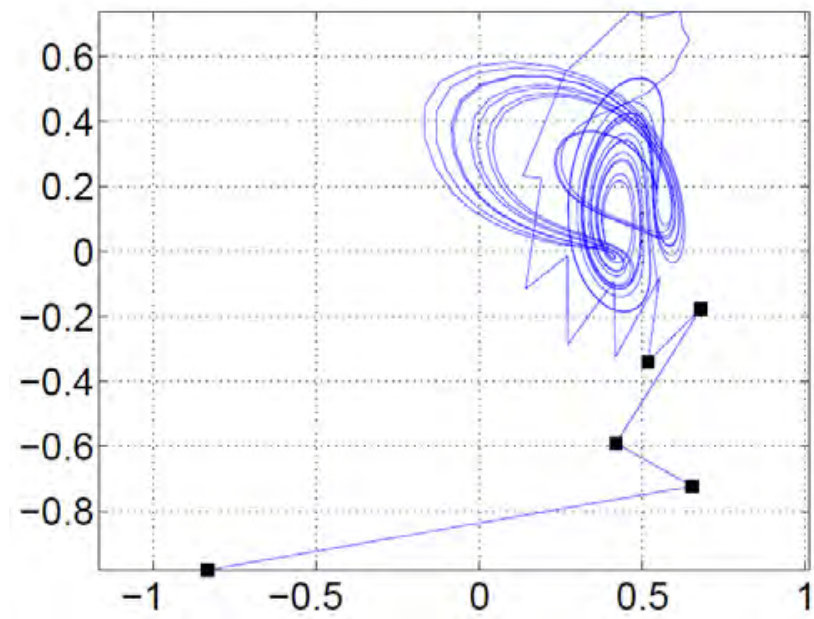

(e) $\{+4,-4,-4\}$

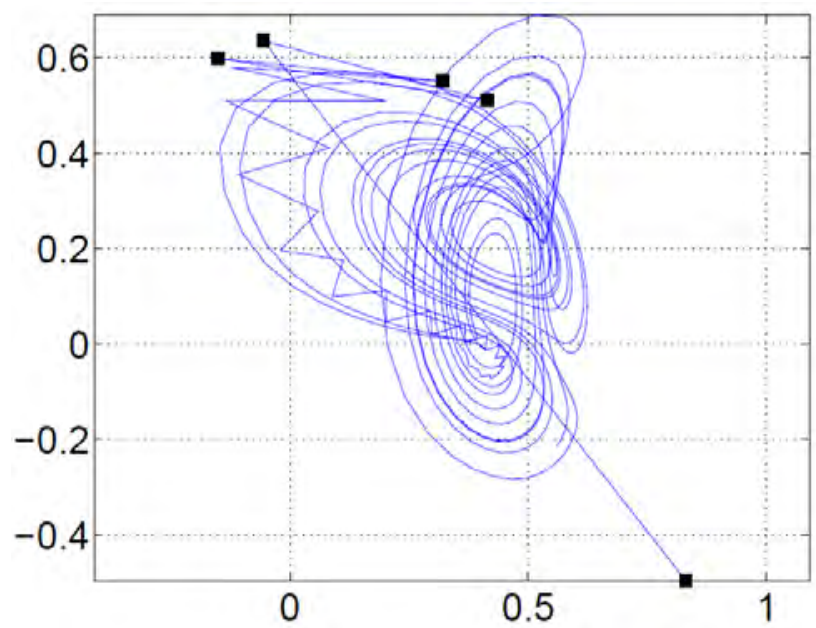

(g) $\{+4,+4,-4\}$

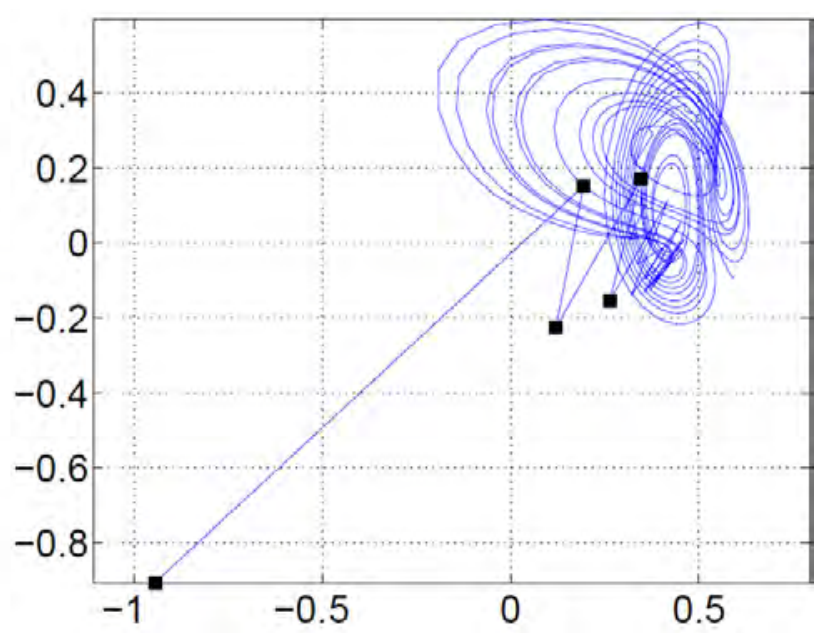

(f) $\{+4,-4,+4\}$

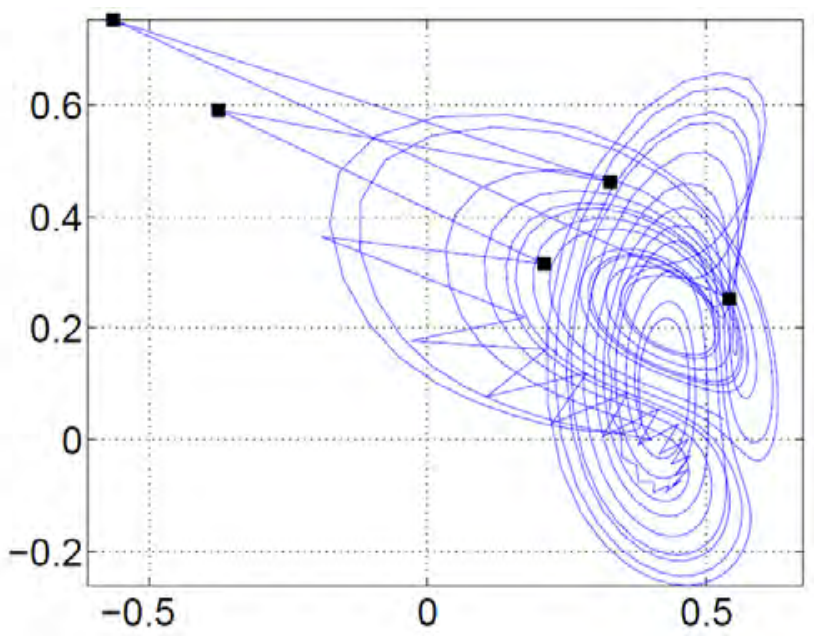

(h) $\{+4,+4,+4\}$

Figure 8. Stability test of a trained SpiralRNN model from simulations with the Lorenz data set. Each sub-figure shows the trajectory of two hidden neurons during autonomous tests with different initial inputs. The starting point coordinates are given beneath each sub-figure. The $\mathrm{X}$-axis and the $\mathrm{Y}$-axis respectively refer to activation values of these two hidden neurons. The first five positions of the trajectory are denoted by black square marks

$$
E_{\text {smape }}=\frac{1}{n} \sum_{t}^{n} \frac{\left|\hat{x}_{t}-x_{t}\right|}{\left(\hat{x}_{t}+x_{t}\right) / 2} \times 100 \%
$$

The competition did not require online learning. Nevertheless, we used this competition to test our approach against sophisticated offline learning methods. As prior knowledge was available through the data sets and as computational complexity was not limited for this application, we have extended our approach in the following way:
- (a) data were rescaled and additional artificial inputs were added providing periodicity information;

- (b) a "committee of experts" was employed in order to reduce the prediction error due to local suboptima;

- (c) the EKF learning process was modified taking into account the SMAPE error function. 
68

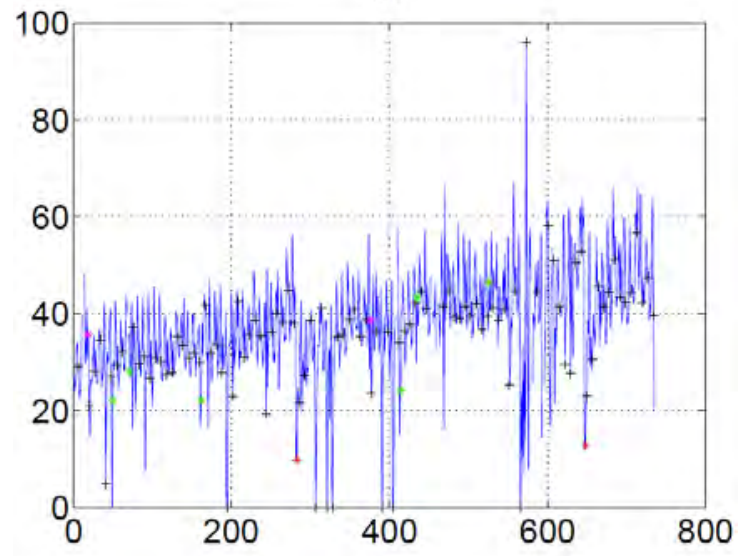

(a) sample 1

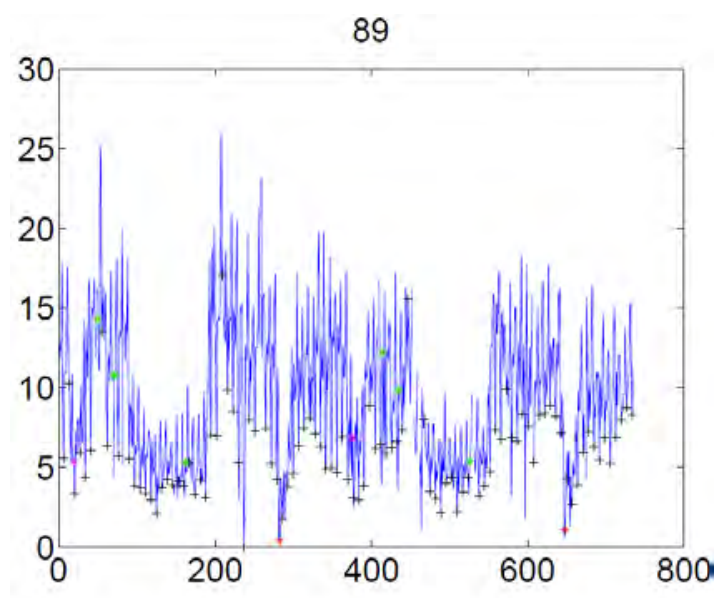

(b) sample 2

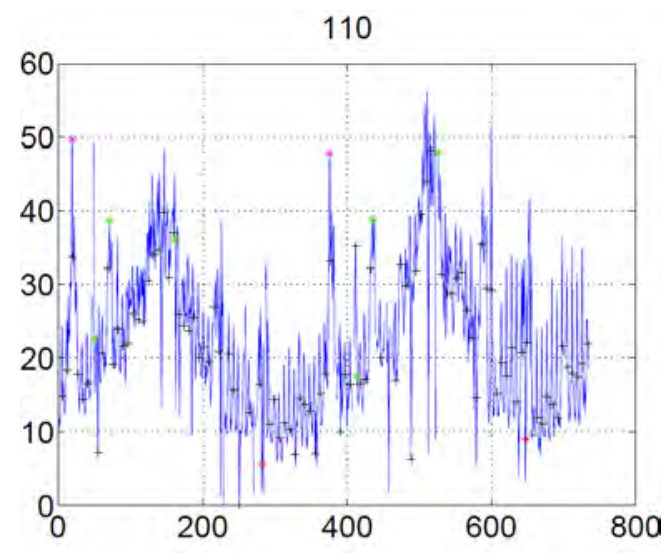

(c) sample 3
1

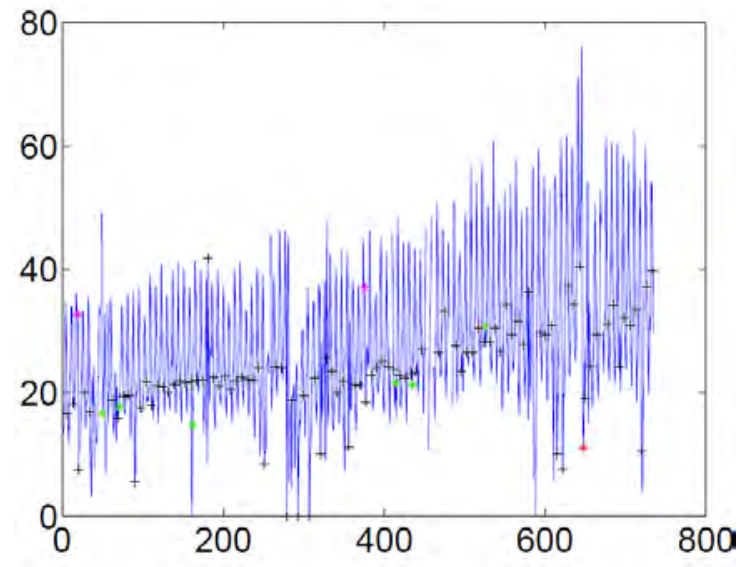

(d) sample 4

Figure 9. Sample data from NN5 competition dataset with cross markers indicating Saturdays

The data presented to the neural network are mapped to a useful range by the logarithm function. In order to avoid singularities due to original zero values, they are replaced with small positive random values. Additional sinusoidal inputs are provided as a representation of calendar information. These additional inputs include:

- Weekly behavior addressing feature $F_{1}$ : Refer to Figure 10(a); the period is equal to 7.

- Christmas and seasonal behavior addressing feature $F_{2}$ and $F_{3}$ : It is often observed from the dataset that, right after the Christmas holiday, withdrawal of money is low and then increases during the year, finally reaching its summit value right before Christmas. Seasonal features do not prevail in the dataset, but they do exist in several of them, e.g. time series 9, 88. As both are regular features with a yearly period, it makes sense to provide an additional input as shown in Figure 10(b) which has period 365.

- Easter holiday bump addressing feature $F_{2}$ : The Easter holidays did not have as much impact on the data dynamics as the Christmas holidays did, but it shows an effect on the usage of ATM in some areas (shown in some time series). Furthermore, as the 58-step prediction interval includes the Easter holidays of year 1998, the prediction over the holiday 
can be improved when the related data behavior is learnt. This additional input uses the Gaussian-distribution-shape curve to emulate the Easter holiday bump as in Figure 10(c).

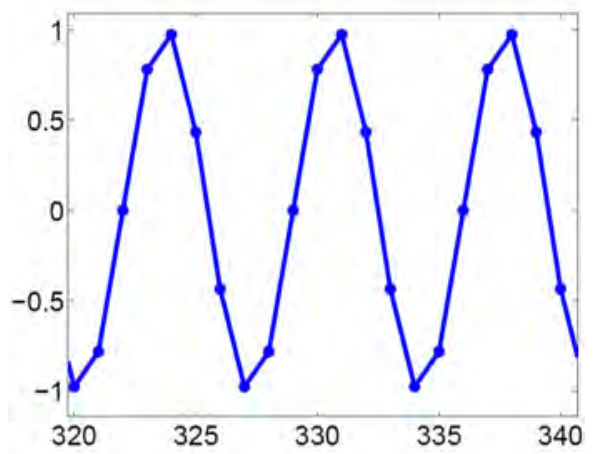

(a) Weekly-input

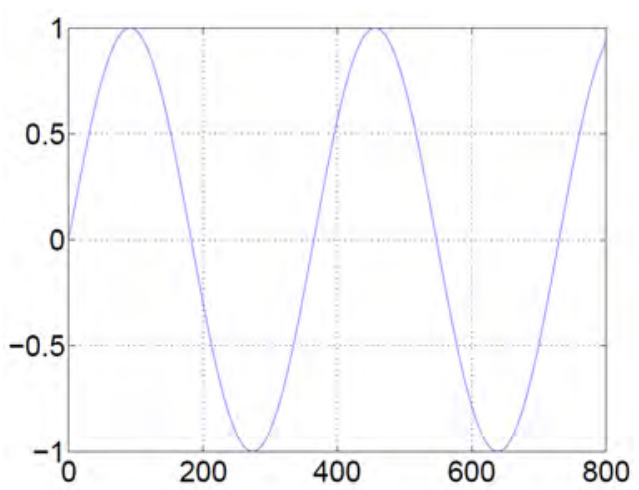

(b) Christmas-input

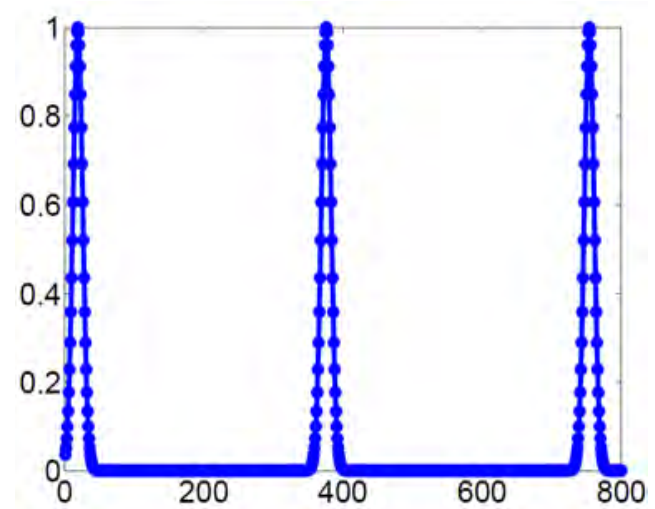

(b) Easter-input

Figure 10. Additional inputs of neural networks. On the $\mathrm{X}$-axis is the time steps, and $\mathrm{Y}$-axis is the additional input value

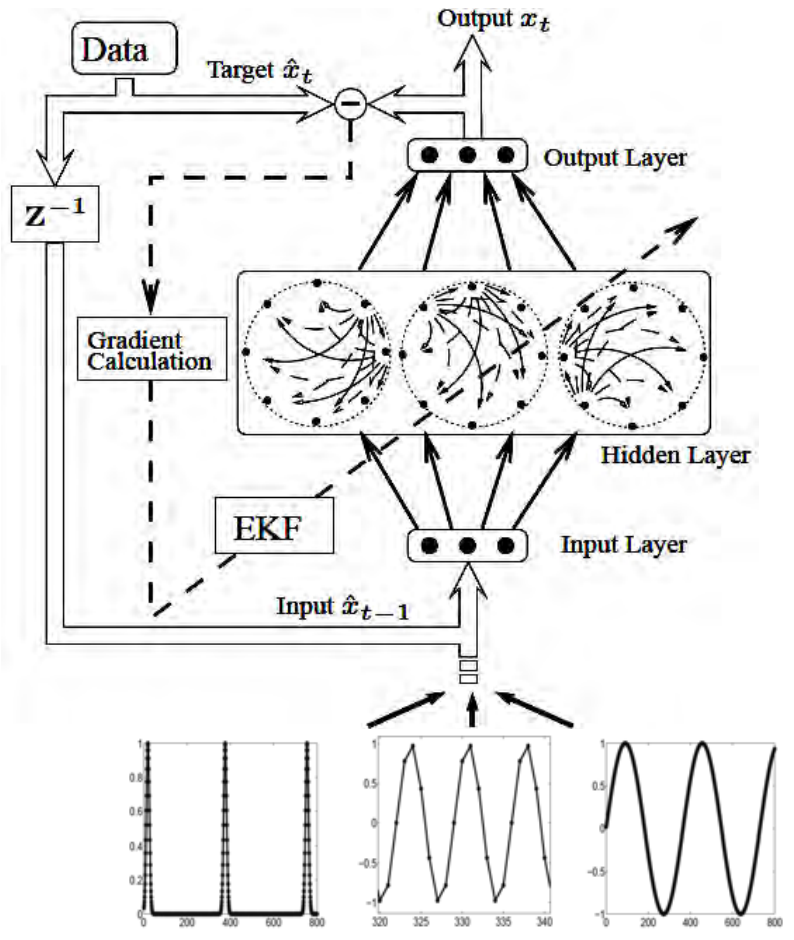

Figure 11. Online training of SpiralRNN in NN5 competition with additional inputs

Supplied with additional inputs, SpiralRNNs were trained online (see Figure 11) such that data were fed-in the network one-by-one and network parameters are trained before the next data is fed-in.

Although SpiralRNN is capable of learning time series prediction, the learned weights usually correspond to local minima of the error landscape as mentioned in [11]. As computational complexity is not an issue for this competition, a committee of experts ansatz is applied.

The committee of experts consists of several SpiralRNN models with identical structure but different initialization of parameter values. Each SpiralRNN model operates in parallel without any interference to the others. They are trained online using teacher forcing. The importance of an expert's output is determined by the average SMAPE value over the last 56 steps. Therefore, at time step $t=679^{1}$, each model $k$ produces a prediction $x_{k, t}$ for the next 56 steps using its output as the input for next time step. After that, online learning continues until the end of the training data. Then the SMAPE error value for the last 56 time steps is calculated according to Equation (15), where $E_{s}$ refers to the SMAPE error.

$$
\varepsilon_{k}=\frac{1}{56} \sum_{t=679}^{56} E_{s}\left(x_{k, t}, \hat{x}_{k, t}\right)
$$

\footnotetext{
${ }^{1}$ The value is calculated via: $t=795-56=679$.
} 
The final prediction for the 56 time steps following $t=$ 735 consists of a weighted average of the committee members' autonomous predictions according to

$$
\begin{gathered}
\varphi=\sum_{k}^{n} 1 / \varepsilon_{k}^{2} \\
\bar{x}_{t}=\frac{1}{\varphi} \sum_{k=1}^{n} x_{t, k} / \varepsilon_{k}^{2}
\end{gathered}
$$

The whole procedure is listed in Table 1, and a schematic diagram Figure 12 depicts the organization of the committee.

As a last step we have to adapt the calculation of the gradient matrix $\Psi$ in Equation (5) to the error function

Table 1. Committee of experts

0. Initialize the $n$ experts;

For a SpiralRNN model $k$, implement online training with the data and make a 56-step

1. prediction $U_{k}$ at time step $t=679$. The prediction value $U_{k}$ will be compared to the data in order to obtain the average SMAPE error value according to Equation (15).

After the prediction at time step $t=679$,

2. continue the online training till the end, and produce another 56-step autonomous prediction $V_{k}$.

3. Based on their $\varepsilon_{k}$ values, combine the prediction $P_{k}$ according to Equation (17).

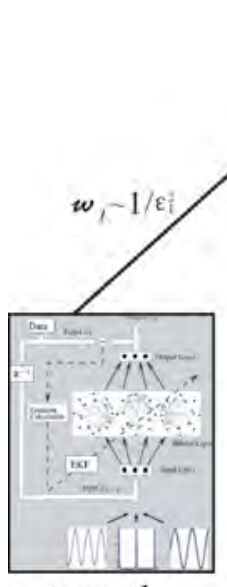

expert 1

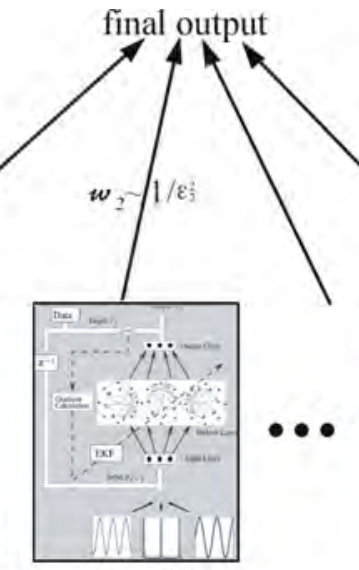

expert 2

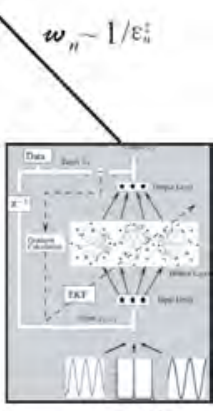

expert 3
Figure 12. Committee of SpiralRNN experts where their weights in committee are determined by their SMAPE values on testing dataset

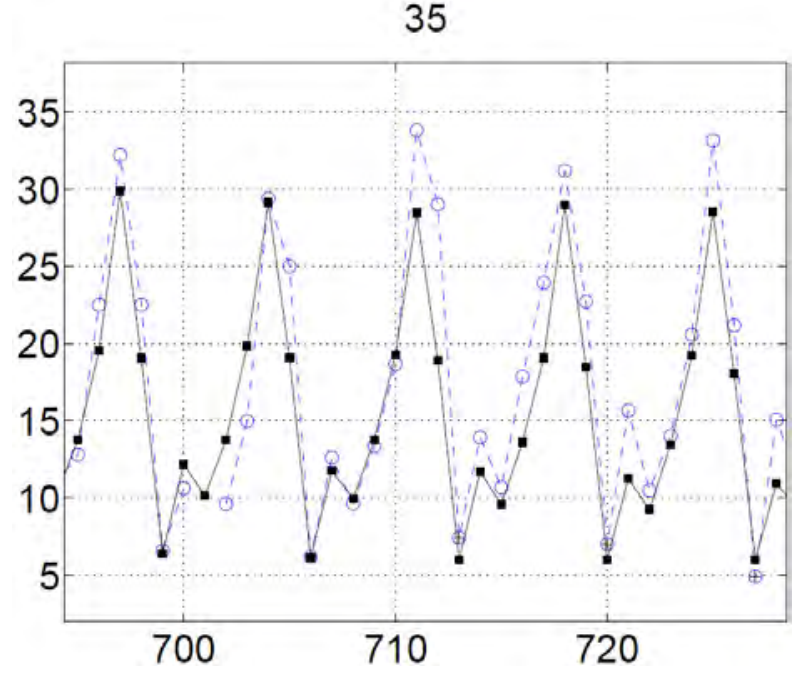

Figure 13. Comparison between result and data showing weekly behavior for time series 35 . Dashed line with circles is the data and solid line with squares is the prediction. The $x$-axis shows the time in days

in Equation (14) according to Equation (20) with $y_{t}=\ln \left(x_{t}\right)$ being the output and $\hat{y}_{t}=\ln \left(\hat{x}_{t}\right)$ the data in logarithmic scale:

$$
\begin{aligned}
S & =\exp \left(y_{t}\right)+\exp \left(\hat{y}_{t}\right) \\
D & =\exp \left(y_{t}\right)-\exp \left(\hat{y}_{t}\right) \\
\Psi_{t} & =-\frac{\exp \left(y_{t}\right)}{S}\left(\operatorname{sign}(D)+\frac{|D|}{S}\right) \frac{\partial y_{t}}{\partial w_{t}}
\end{aligned}
$$

For data sets with missing values we replace these with predicted values and simultaneously skip the weight update while still aggregating the gradient.

We have evaluated our approach using the last 56 data of the available data while the remaining data were used for online-training. In Figure 13, prediction and data are compared for time series 35 which has a pronounced weekly periodicity. Obviously, this periodicity can be reproduced pretty well, even details like the small bump.

Seasonal behavior of data can also be learned as shown in Figure 14. The curves in both sub-plots begin with the values in Christmas holidays (with time indices around 280 and 650). The rise and fall of the data during the first about 100 days and a subsequent rise in Figure 14(a) can also be seen one year later in Figure 14(b). Obviously, the model is able to capture this behaviour pretty well. 
Easter holidays can also be recognized by the trained model as shown in Figure 15 for time series 110. Since the expert-weight in the committee is determined by a

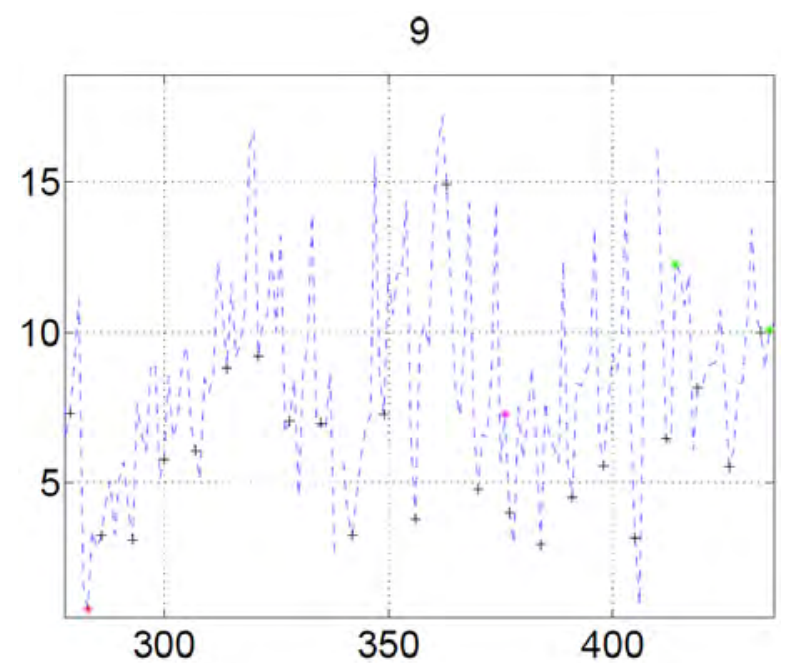

(a) seasonal-data previous year

9

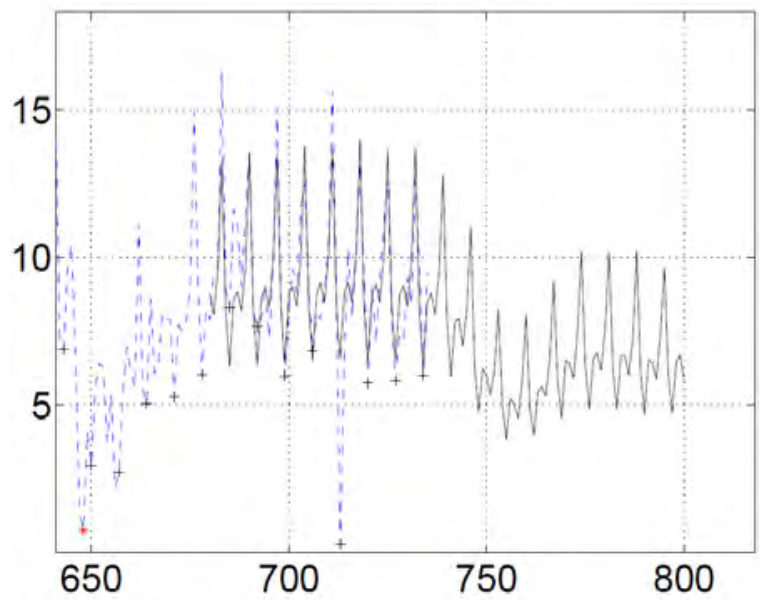

(b) seasonal-data and prediction

Figure 14. Comparison between prediction and data for time series 9 showing seasonal behavior. Dashed curve is the data and solid curve is the prediction. The $x$-axis denotes time measured in days. The upper figure shows the same period one year before

Table 2. Statistic results. The committee-averaged SMAPE error value of the expert committee on all 111 time series with varying number of experts

\begin{tabular}{|c|c|c|c|c|c|c|}
\hline experts & 3 & 5 & 10 & 15 & 20 & 30 \\
\hline \hline SMAPE & 21.4 & 20.9 & 21.4 & 22.3 & 22.2 & 21.6 \\
\hline
\end{tabular}

97

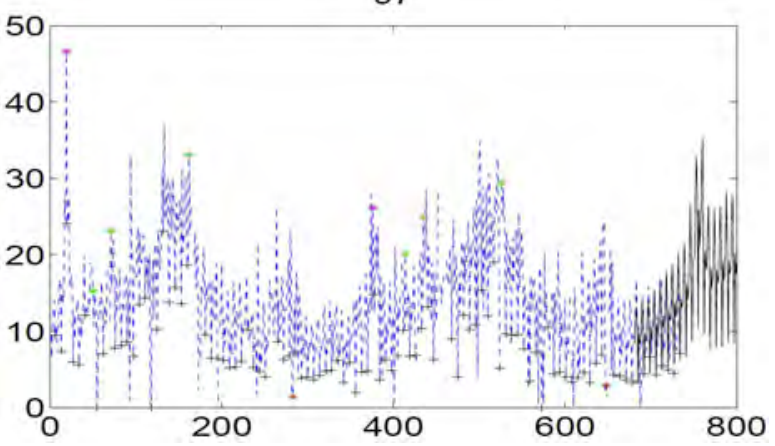

Figure 15. Prediction result (solid curve) and data (dashed curve) for time series 110 showing a peak around Easter. The $x$-axis displays time in days

period not containing Easter holidays, the prediction for them might be expected to be less accurate. Nevertheless, as shown in Figure 15, the corresponding bump in the data has been learned well. The Easter holidays in 1996 to 1998 occur at times around 20, 375 and 755. In Figure 15, the prediction for the Easter holidays 1998 shows a similar behaviour as the Easter holidays in 1996 and 1997 with a pronounced peak.

Table 2 shows the SMAPE errors on the test set (i.e. the data from the last 56 time steps) for a varying number of experts. Obviously, the number of experts has no significant influence on the average result. This indicates that the learning process is quite reliable; in addition this allows saving computational effort using a small committee. Figure 16 shows the histogram of committeeaveraged SMAPE values over the 111 time series in the dataset. The majority of the results have a SMAPE value around 20.

Our approach has won the time series forecasting competition at WCCI2008 ${ }^{1}$ for both the full data set and a reduced data set consisting of 11 time series. Among the competitors was also commercial software currently implemented in the ATMs. Although a few sophisticated statistical and neural network approaches which were not taken into account for the awards performed better, the final results for the top 10 performing approaches are quite close. The most important result for our approach is that even with a remarkably simple extension our online learning approach is competitive to world class statistical and neural network solutions.

\subsection{Mouse Tracking - A Toy Example}

Besides using artificial data we also made experiments

${ }^{1}$ http://www.wcci2008.org/. 
with real-life data. For this purpose we recorded the position of a computer mouse moved along a periodic trajectory by a person and trained a SpiralRNN online with these data. The background for this experiment is e.g. applications in logistics and factory automation where the motion of transported goods or robot arms has to be tracked and analyzed.

For the mouse tracking we did some preprocessing on the data in order to become independent of the velocity of the mouse. For this purpose we took the path-length of the trajectory as the "time"-variable and interpolated the position at equidistant length intervals. These positions after a suitable rescaling - are used as input for the Spiral RNN during online training, trying to learn to predict the next position on this discretized trajectory. Figure 17 illustrates such discretization. Figure 18 shows one particular trial. The blue line is generated by a user moving the mouse along a lying figure 8. After about thirteen iterations of this pattern the online training is stopped and the Spiral RNN is fed with its previous output thus generating the red trajectory. Obviously, the Spiral RNN has learned to reproduce the full trajectory. The mean square error (MSE) is normalized to the variance of the time series. The reader may notice that this pattern is nontrivial in the sense that a certain amount of short-term memory is necessary in order to select the correct direction at the crossing.

Such a pattern produces 2-dimensional periodical time series as targets for the neural networks. For this example we have also analyzed the frequency spectrum of the data and the predicted outputs of different neural network models. The result is given in Figure 19 with blue dashed and green solid lines for the two time series respectively. SpiralRNN produces (refer to Figure 19(b)) autonomous output trajectories with a frequency spectrum similar to the frequency spectrum of the target data

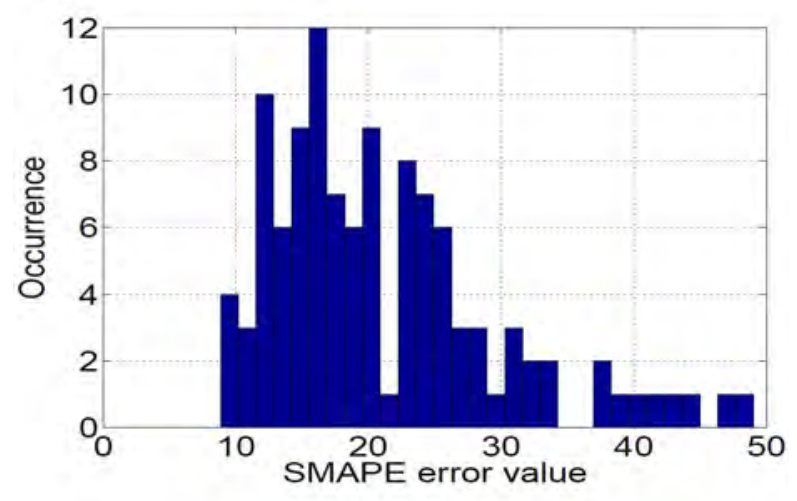

Figure 16. Histogram (over 111 time series) of committeeaverage SMAPE error

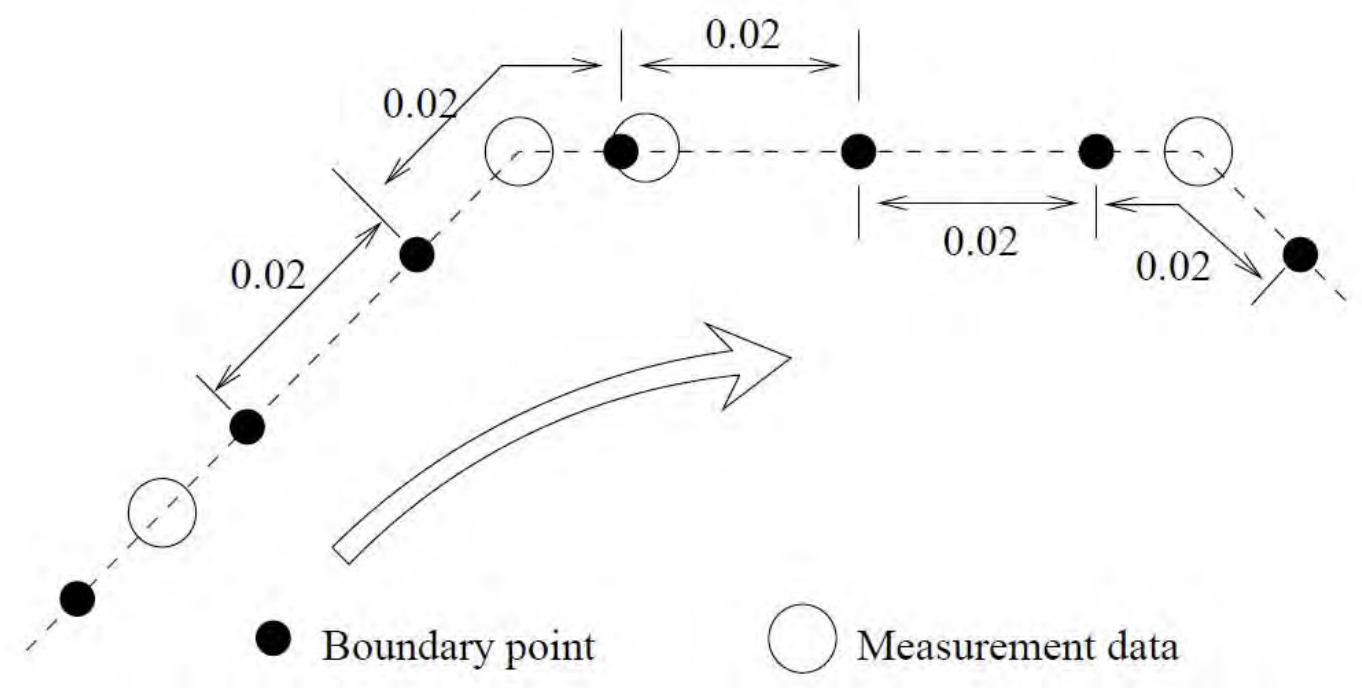

Figure 17. Segmentation by boundary points in MouseTracking. The hollowed arrow indicates the movement direction, the dashed line is the movement path of the cursor, circles represent the position measured by computer in every 1/100 second, and black dots refer to boundary points that segment the movement path of cursor. The identical length between boundary points is “ 0.02 ". The positions of these boundary points are taken as the training data at each "time” step 

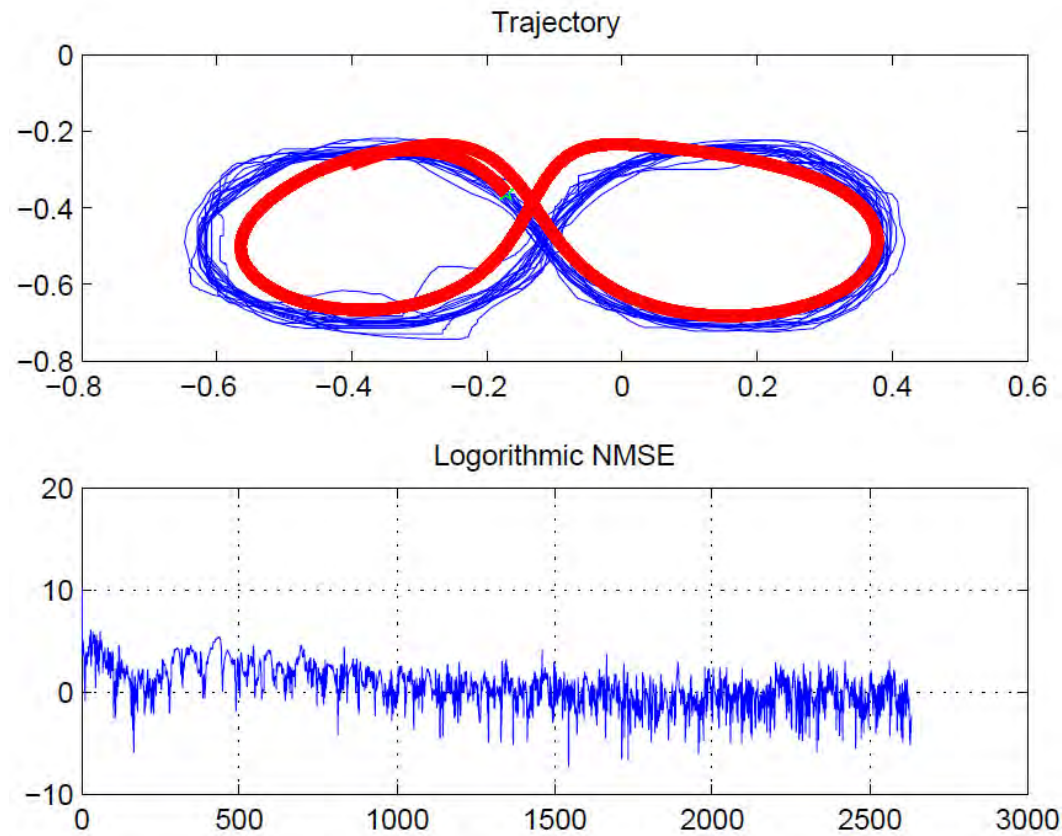

Figure 18. Mouse tracking example: The noisy line in the upper figure is the trajectory generated by the user; the smooth line is the test with the Spiral RNN trained online and iterated with its own output as next input. The lower figure displays the logarithm of the normalized one-step prediction mean square error which decays along training

(refer to Figure 19(a)), while the other models cannot.

If the training time was too short, the network usually runs into the closest of a set of fixed points; two of them are usually located within the two loops of the Figure eight. This experience is confirmed with more complex pattern where the required training time increases correspondingly. One reason is the usually large amount of

frequency analysis of original trajectory

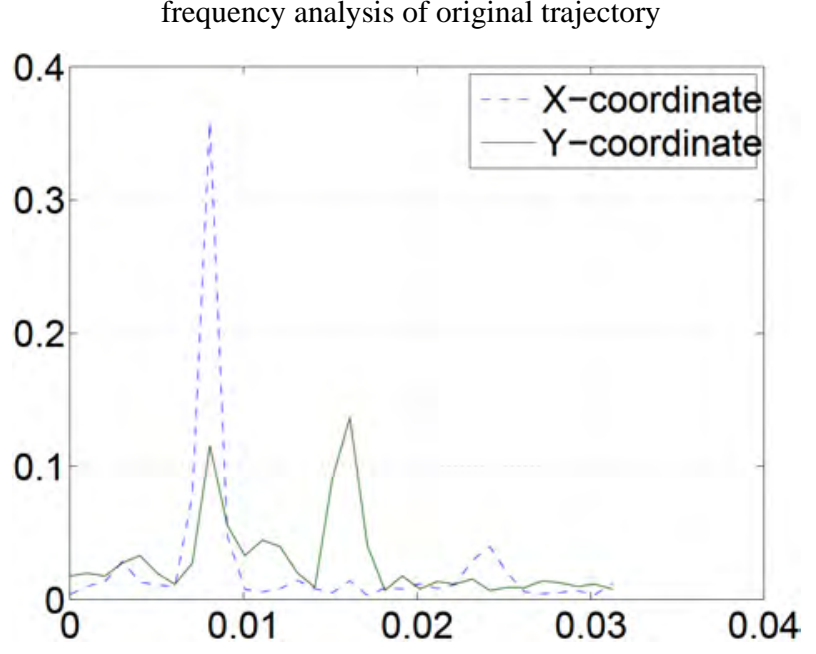

(a) Target noise due to manually moving the mouse. This noise prevents establishing a large enough short-term memory which may be required for correct prediction and favors the development of fixed-point attractors.

Sharp turns in a trajectory are also difficult to reproduce. Typical examples are triangles or squares. Even if the network has learned to reproduce such a pattern, it

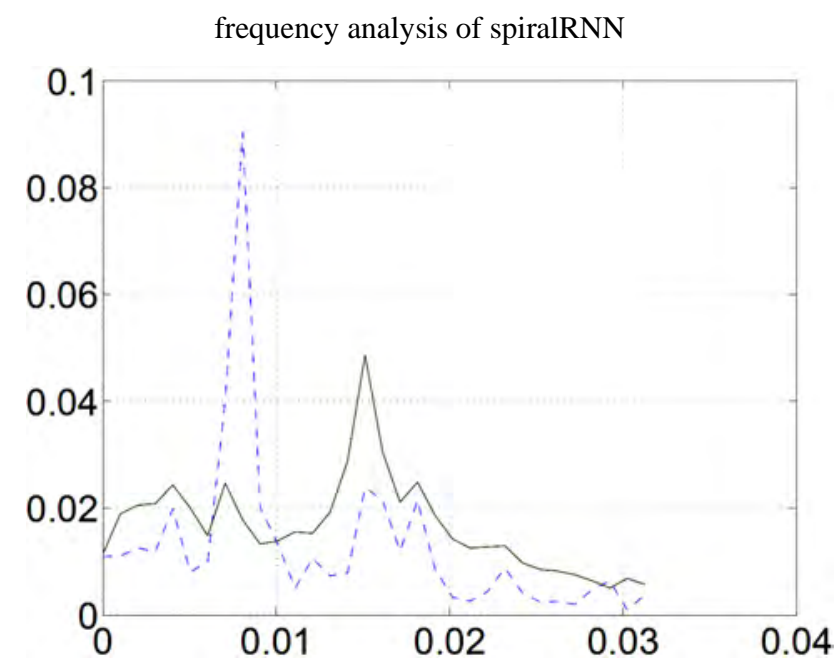

(b) SpiralRNN 
frequency analysis of ESN

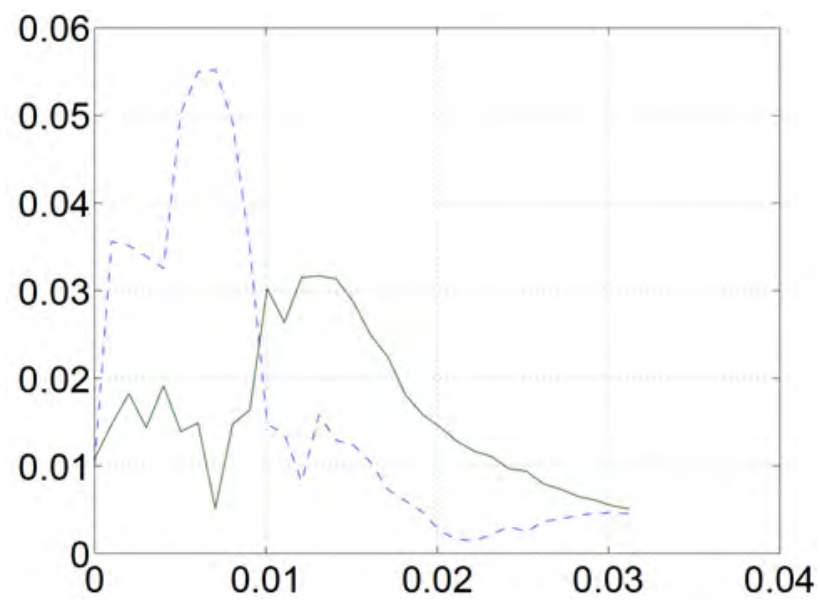

(c) $E S N$ frequency analysis of BDRNN

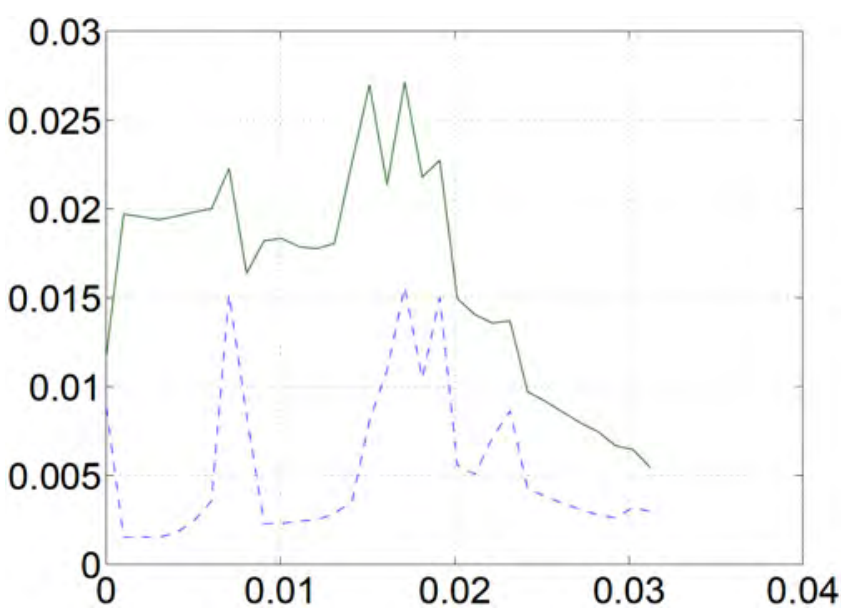

(d) BDRNN

Figure 19. Frequency analysis of the output trajectory after 5000 training steps of different neural network models with around 100 parameters. Note that the vector value at frequency "zero" is trivial and is therefore omitted in diagrams, and that the period length of data is $\mathbf{1 3 0}$ therefore the dominant frequency is $\frac{1}{130} \approx 0.008$

always produces smooth turns and it takes much more additional training to sharpen these turns.

\subsection{Duty Cycle Adaptation in Wireless Sensor Networks}

In energy autarkic wireless sensor networks, achieving a long lifetime requires the wireless sensors to switch off individual components as often as possible. Most energy is used by the transceiver in transmit mode as well as in receive mode. Therefore, sensor nodes have organized their activity in duty cycles consisting of an active period and a passive period with most components switched off. The longer the second passive period is, the smaller is the duty cycle and the longer is the lifetime of the sensor node.

One way of reducing the duty cycle effectively is to skip listening to neighboring sensor nodes for their data and instead use predicted values. For this reason, a sensor node does not have to transmit its own sensor values to its neighbors as well. This implies that sensor nodes can increase their inactivity period thus reducing the duty cycle. Of course, the prediction error will increase also with decreasing duty cycles. Therefore, there will be an optimal choice concerning prediction error and energy saving.

We analyzed this application example using a simulation of a room equipped with 10 temperature sensor nodes and 4 heat sources as shown in Figure 20. The varying strength of the heat sources was modeled by a 4-dim. chaotic Lorenz time series with 6000 time steps; a short interval is shown in Figure 21. The diffusion of heat from the heat sources into the room was modeled by a simple diffusion model, discretized in space and time, with random additive noise. An example for the resulting sensor values is shown in Figure 22.

Each of the 10 sensor nodes is equipped with a SpiralRNN supposed to predict the sensor node's measured temperature value and those of the neighboring nodes. The SpiralRNN had 100 trainable weights for this evaluation. The neighbor nodes are those nodes which have a direct communication link to the sensor node (see Figure 20). In regular intervals, the sensor nodes broadcast their current sensor value to their neighbors. One complete exchange involving all 10 sensor nodes corresponds to one time step in the simulation.

In order to reduce the duty cycle, the sensor nodes do not listen to their neighbors every second time step. This saves energy for communication: with a normal duty cycle, a sensor node with $k$ neighbors has to run its transceiver $2^{*}(k+1)$ times in two intervals; for a reduced duty cycle it is $2+k$ times in two intervals, a reduction by a factor of $\frac{2+k}{2+2 k}$. With just one neighbor this is a factor of 


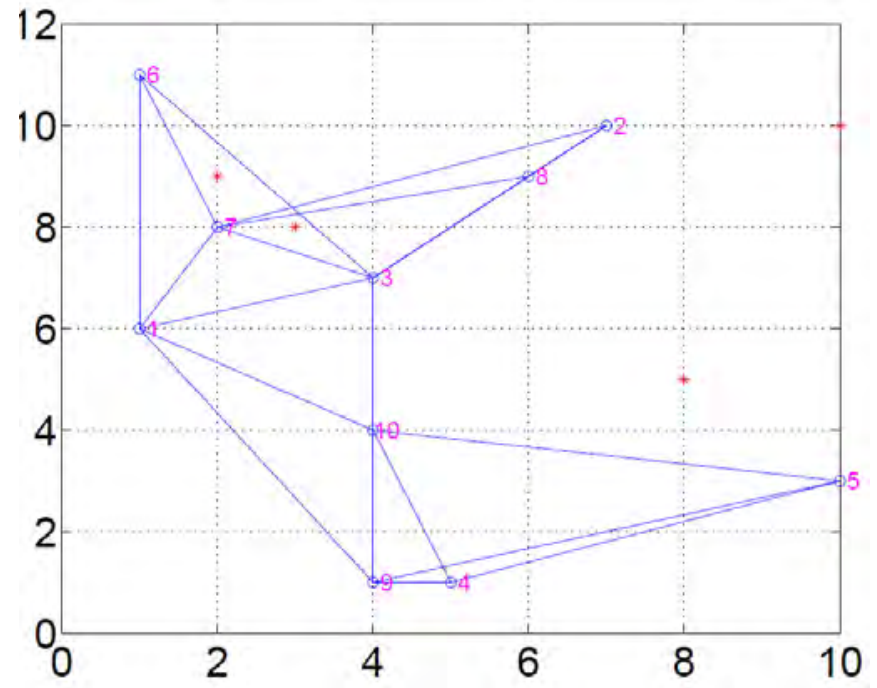

Figure 20. Simulation environment with sensors (numbered circles) and heat sources (stars). Also shown are the communication links between sensor nodes

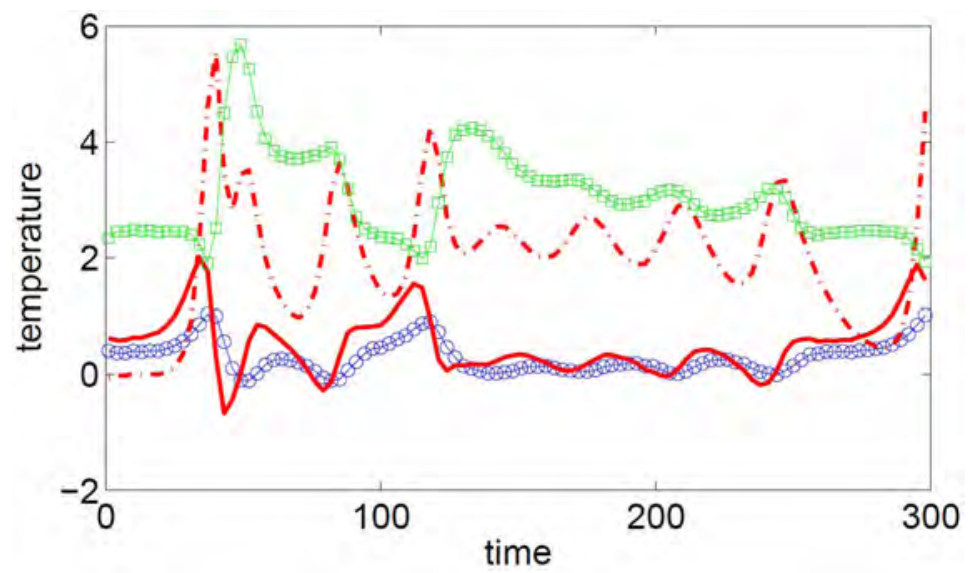

Figure 21. Example of time series of heat sources. Only one period is shown. Similar pattern occur in other periods

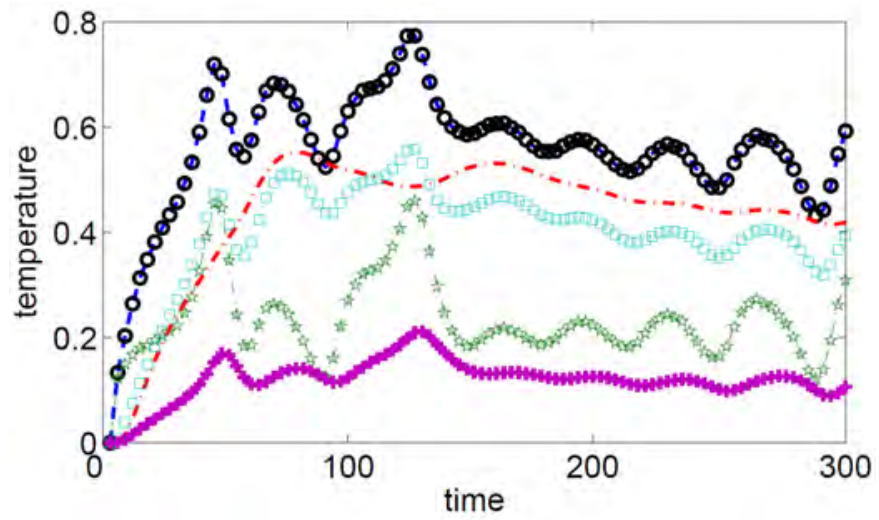

Figure 22. Example of measured temperature values at the different sensors. Only one period is shown. Similar pattern occur in other periods. 


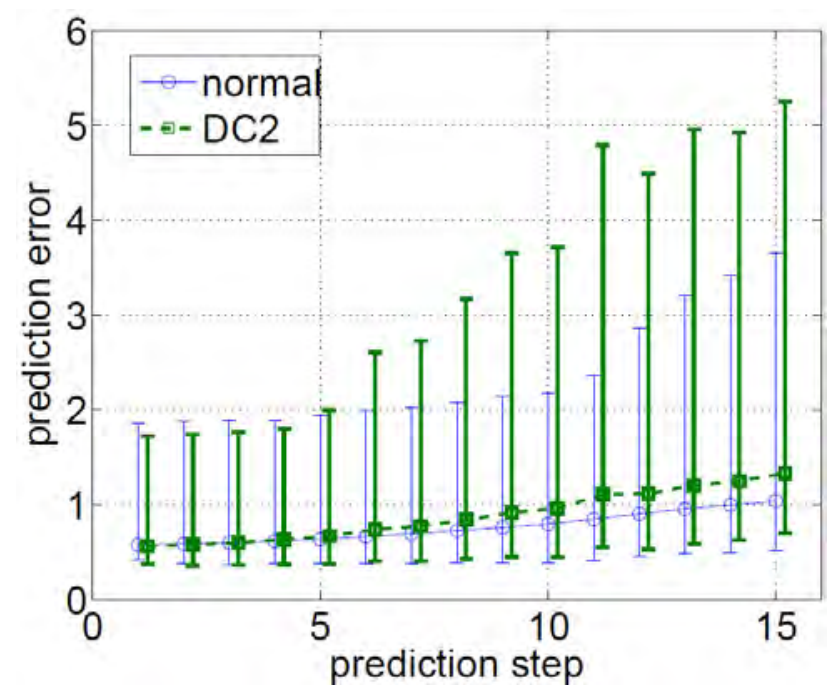

Figure 23. Comparison of relative prediction error for normal duty cycle (continuous line, circles) and reduced duty cycle (dotted line, squares, listening only every second interval). Shown are mean, minimum and maximum of the rms error of 30 runs

0.75 , with 4 neighbors it is a factor of 0.6 , with 9 neighbors already 0.55 and quite close to the large- $k$ limit of 0.5 .

However, the picture is not complete without looking at the accuracy of the predicted instead of measured sensor values. We performed 30 independent runs with randomly chosen locations of sensors and heat sources, random scaling of each of the heat sources with a factor in the range $[0,1]$ and additive noise of amplitude 0.01 at each update of the heat diffusion on every grid point. The result for the relative error - normalized for each sensor to the variance of its sensor value time series - is shown in Figure 23: For up to 5 prediction steps the performance with the reduced duty cycle is almost the same as for the normal duty cycle. The outliers with a normalized error larger than one are due to sensors with an almost constant sensor value whose variation is mainly due to noise. This result indicates that there is even more room for duty cycle reduction for this example: Listening to the neighbors only every fourth interval yields a duty cycle reduction of $\frac{4+k}{4+4 k}$; with 4 neighbors this gives a reduction factor of 0.4 , with 9 neighbors it gives 0.325 and the large- $k$ limit is 0.25 .

\section{Conditional Prediction}

So far, SpiralRNN turned out to be excellent in online learning the dynamics of stationary processes. However, in reality we often have to deal with non-stationary processes. A typical example are cars approaching a crossing: without knowledge about their destination one usually can predict their approaching the crossing and their leaving again, but not whether and into which direction they turn at the crossing. Even our daily activities consist of sequences of typical pattern like walking, standing, talking, eating, and drinking and so on. Again, without additional knowledge about the motivation of a person usually it is impossible to predict the sequence of these patterns, which can themselves be predicted very well.

\subsection{Application Example: Warehouse Logistics}

We consider an application example from the logistics domain: A warehouse stores goods of different type in different areas (refer to Figure 24). Newly delivered goods have to be transported to these areas by trucks; we assume that these trucks always use the same trajectory to a specific destination. Each truck is equipped with a SpiralRNN learning the different trajectories. However, different from previous examples we assume that there is additional information available about the type of product; for example, this could be provided by a RFID reader at the entrance registering newly delivered goods carrying RFID tags with product information. After having learned the trajectories accompanied with this additional information, the Spiral RNN just receives this additional information and has to recall the trajectory. This can be used for diagnosis purposes (e.g. is it the correct trajectory?) or for autonomous truck driving. 
In addition to the current position coordinates, the input vector of the Spiral RNN now also stores an additional trigger signal [12]. The network output is trained to predict the changes in this vector. At occurrence of each trigger signal, the hidden state of the network is reset to zero and the input vector consists of some arbitrary, but otherwise fixed position vector and of the trigger signal; the latter is kept at this initial value until an- other trigger signal indicates completion of the trajectory or start of a new trajectory. At subsequent steps, the network - while in online learning mode - learns to predict the changes in position and change in the trigger signal; the latter is actually zero. In recall mode, the previous output added to the previous input together representing the new position and trigger signal is fed back as input for the next time step.

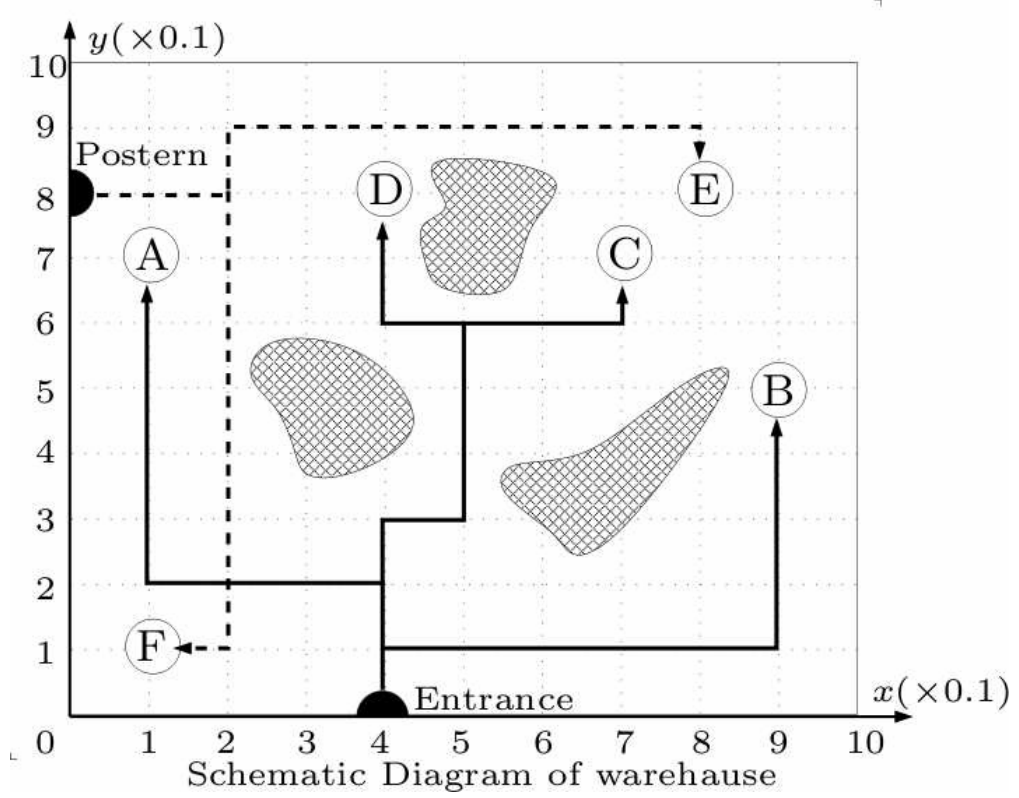

Figure 24. Application example "warehouse": Depending on their type, newly delivered goods have to be stored in different areas labeled by A-F. The trajectories used by trucks are shown as well

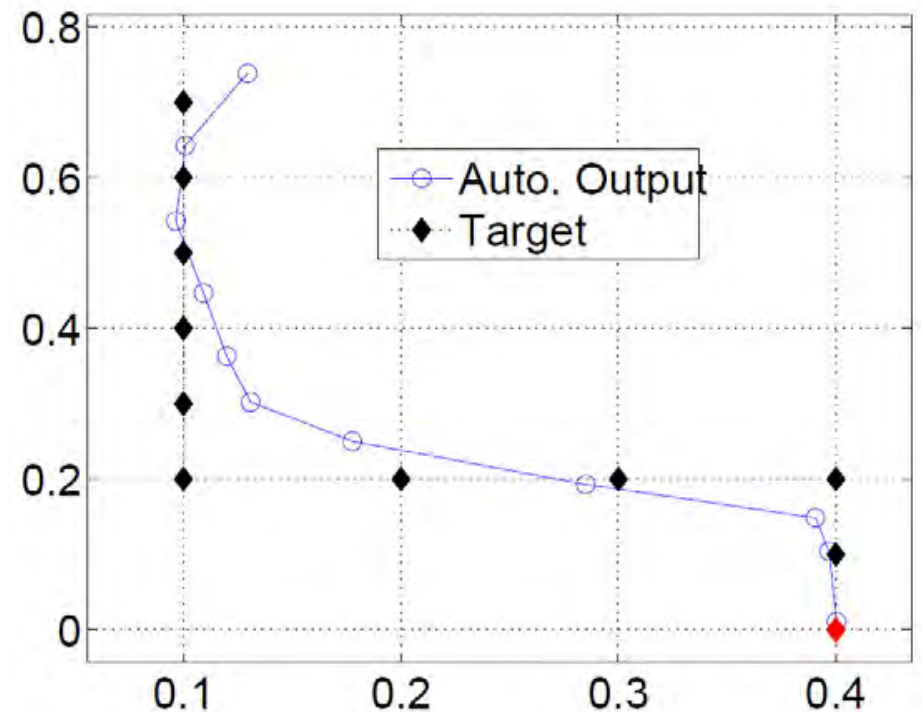

Figure 25. Conditional prediction of trajectory to area A (circles). The starting point of the trajectory is at [0.4,0]. 


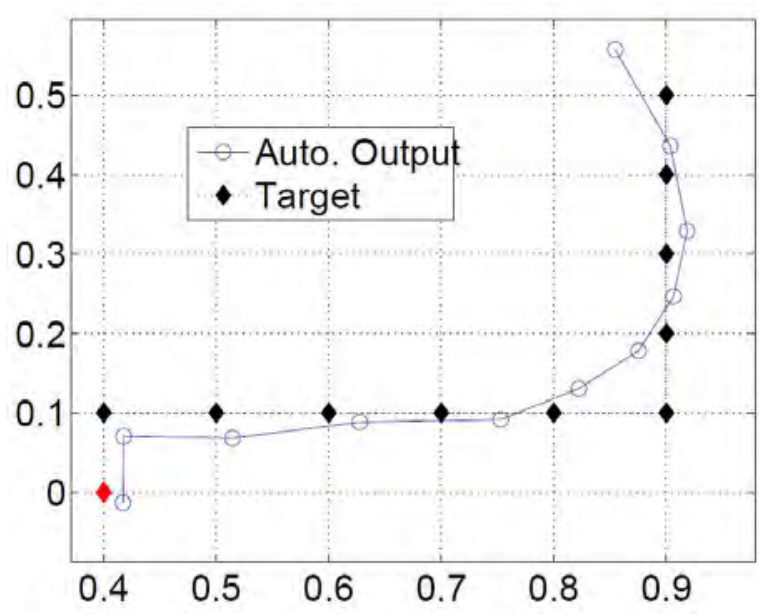

Figure 26. Conditional prediction of trajectory to area $B$ (circles). The starting point of the trajectory is at [0.4,0].

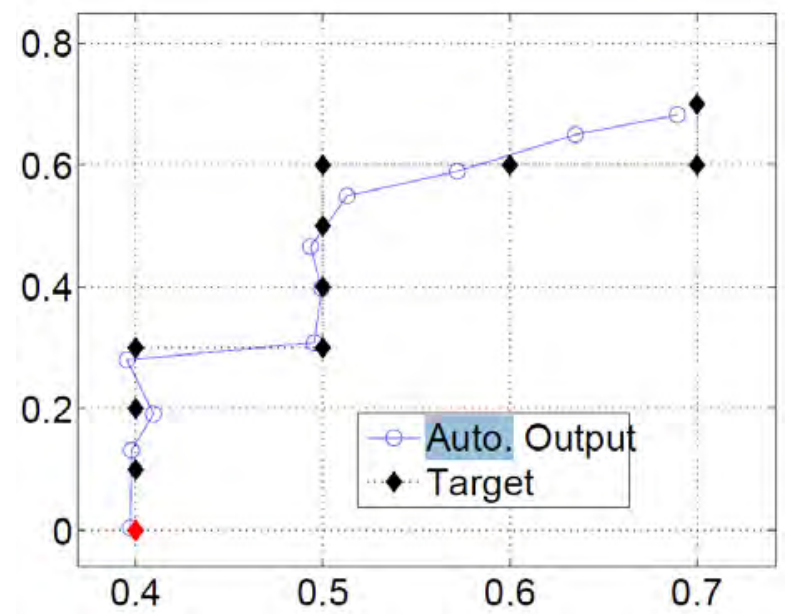

Figure 27. Conditional prediction of trajectory to area $C$ (circles). The starting point of the trajectory is at $[0.4,0]$.

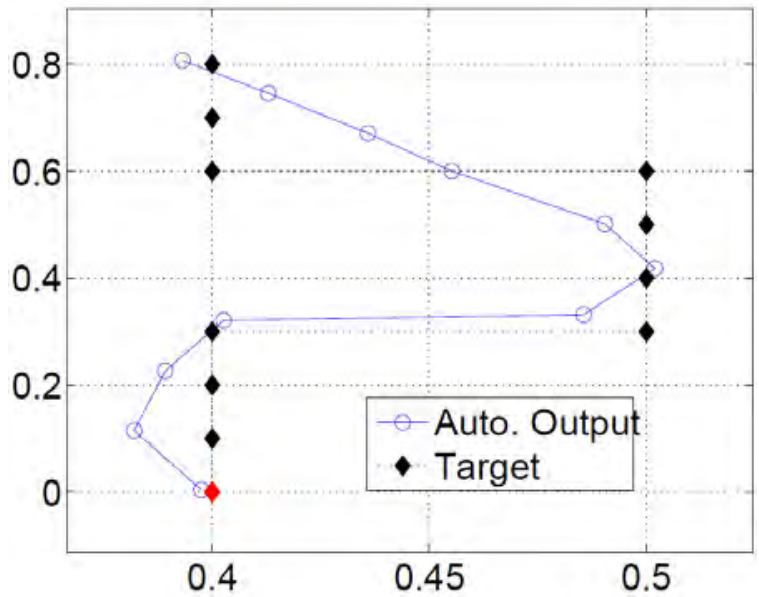

Figure 28. Conditional prediction of trajectory to area $D$ (circles). The starting point of the trajectory is at [0.4,0]. 
Figures 25-28 show the performance of a trained SpiralRNN on reproducing the trajectories to the areas $\mathrm{A}, \mathrm{B}$, $\mathrm{C}$ and $\mathrm{D}$ given just the initial trigger signal. The network had 200 trainable weights and each trajectory was presented 20 times until the root mean square error (RMSE) for each trajectory was below a threshold of 0.05 , i.e. half grid spacing. Obviously, the trajectories have been learned pretty well.

\subsection{Storage Capacity}

Encouraged by the good performance of this approach, we investigated the storage capacity of SpiralRNN in dependence on the number of hidden neurons and the length of the pattern time series. For this purpose we created random two-dimensional trajectories with each a different but randomly chosen trigger signal value. We considered trajectories of 6 and 11 steps. The performance of a trajectory prediction is evaluated as above as the MSE over the whole trajectory. We consider a number of trajectories trainable if within at most 30 trials with each consisting of 50 presentations of each trajectory the RMSE over all trajectories is at least once below the threshold of 0.05 . The whole process contains 5 steps, as the following:

1) The experiment starts with small networks (The value of $n_{h}$ starts from 6 , such that the network comprises 3 hidden units and each hidden unit has 2 hidden nodes); Training data is the combination of $n_{p}=2$ trajectory patterns.

2) With each pair of $\left\{n_{h}, n_{p}\right\}$, at most 30 simulations are performed, each of which contains 50 training rounds. In each training round, the learning model is trained on-line with the presentation of these $n_{p}$ trajectory patterns, in a shuffled sequence but each pattern only once. The hidden-state vector of network is reset before the training with any trajectory pattern starts. After every 10 training rounds, a testing round starts, trying to reproduce all $n_{p}$ trajectories in an autonomous manner separately.

3) The autonomous output results are evaluated. Let $l_{p}$ be the length of trajectory patterns (the $l_{p}$ value is identical to all pattern), $x_{i, k}$ be the $i^{\text {th }}$-step ahead prediction over the $k^{\text {th }}$ pattern, and $\hat{x}_{i, k}$ be the respective target, the evaluation error $\varepsilon$ is calculated as:

$$
\varepsilon \varepsilon=\frac{1}{n_{p}} \frac{1}{l_{p}} \sum_{i=1}^{l_{p}} \sum_{k=1}^{n_{p}}\left(\hat{x}_{i, k}-x_{i, k}\right)^{2}
$$

4) If it satisfies $\varepsilon \leq 0.05^{2}$, this particular SpiralRNN network with $n_{h}$ hidden nodes is claimed to be capable of reproducing $n_{p}$ number of trajectory patterns of length $l_{p}$, and thus the associative memory satisfies $\mathrm{M}_{a} \geq n_{p}$. The model (with the same structure but re- initialized values) will then be assessed with a training data with more patterns, i.e. higher $n_{p}$ value.

If $\varepsilon \leq 0.05^{2}$ is not satisfied at least once within 30 simulations, it is claimed that SpiralRNN with $n_{h}$ hidden nodes is not able to reproduce $n_{p}$ number of trajectory patterns with length $l_{p}$. New experiment will start with same value in $n_{p}$ but a larger SpiralRNN model by increasing the $n_{h}$ value by 3 . Note that the threshold value is set to 0.05 , half the grid distance 0.1 , so that the prediction can be rounded to the nearest grid point if the error is less than 0.05 .

5) The experiment are continued until simulations with $n_{h}=60$ are finished.

Figures 29 and 30 show the dependence of the number of trainable pattern on the number of hidden neurons. Obviously, the graphs show an almost linear dependence. For short pattern of length 6 we get a relation $n_{p} / n_{h}=$ $0.64 \pm 0.04$ and for long pattern of length 11 we get $n_{p}$ $/ n_{h}=0.31 \pm 0.02$.

\section{Tiny OS Implementation}

Finally we report on an implementation of SpiralRNN in a typical wireless sensor platform. One example of such a sensor platform is the TelosB[13] sensor mote from the University of California at Berkley. It provides sensors for temperature, light and humidity. A microprocessor (Texas Instruments, MSP430, 16 bit, 8 MHz, 48kB ROM, 10kB RAM) allows to run software for communication, data processing and energy management. A wireless transceiver (Chipcon 2420, IEEE802.15.4, 2.4 GHz, 250 kbps) provides communication links to other sensor motes or to a gateway. 


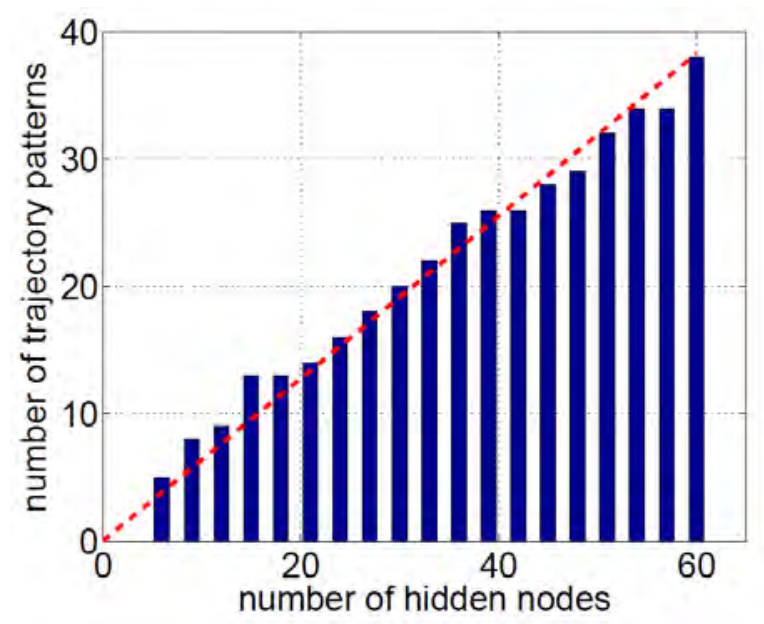

Figure 29. Pattern storage capacity of SpiralRNN with different number of hidden nodes and with patterns of length 6

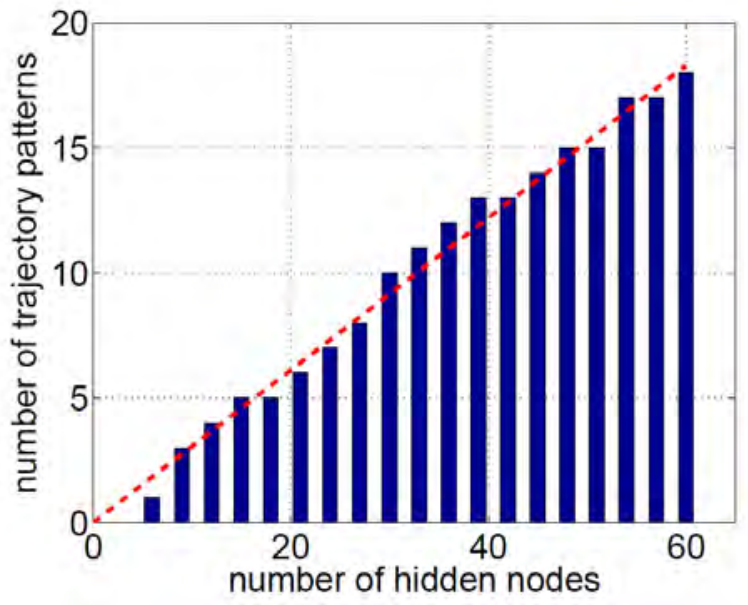

Figure 30. Pattern storage capacity of SpiralRNN with different number of hidden nodes and with patterns of length 11

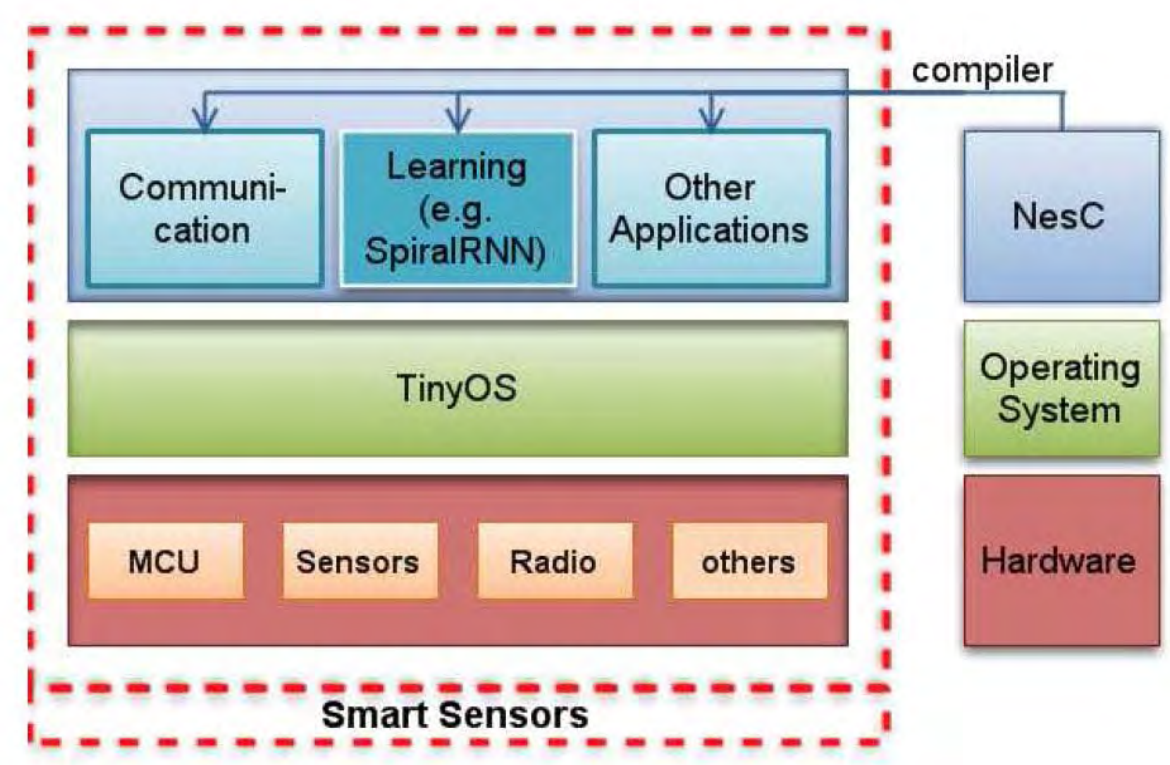

Figure 31. Structure of a typical smart sensor mote. TinyOS is the specific operating system for embedded system such as smart sensor and provides interfaces among hardwares and softwares. NesC language, as a compiler, compiles and builds softwares for different applications

We have deployed TinyOS, a small footprint operating system developed at the University Berkeley, on such motes. Programs developed for TinyOS are written in the programming language NesC [13]. Figure 31 shows the typical software architecture of a sensor mote. It allows implementing even advanced functionalities like analysis and learning on such embedded devices.

In order to accelerate the task execution, we replaced the floating point operations by suitable fix-point opera- tions. Thus we achieved a runtime for one online learning iteration of the SpiralRNN with a single input/output node and 6 hidden nodes (totally 24 parameters in the neural network) of roughly 300 milliseconds, a reduction of about a factor 8 compared to a floating point arithmetics implementation. Figure 32 shows a comparison of the floating-point model and the fixed-point model with respect to processing time and memory requirement (RAM) for a TelosB sensor mote (both models have sin- 
gle input and output neuron). The limited memory capacity (10 Kbytes) of such a sensor mote prevents deployment of floating-point model images with more than 36 parameters or running it with more than 32 parameters. In contrast, the fixed-point model takes less than one second for each online learning step for networks with up to 40 weight parameters (i.e. 10 hidden nodes). Similarly, the fixed-point model requires less RAM allowing working with more complex neural networks.

It is also interesting to compare the processing time of the pure feed-forward step with the complete online learning step. Figure 33 compares two fixed-point models with feed-forwarding (FFD) only and with full online learning (FULL) including feed-forwarding, gradient calculation and extended Kalman filter update. Obviously, the full online learning step requires much more time; while a pure feed-forward step requires only $11 \mathrm{~ms}$ for 48 weight parameters, the full online learning step takes about $1500 \mathrm{~ms}$ for the same network. As a consequence, one can reduce the number of learning steps when the neural network is well trained and thus save quite some energy.

We close this section with a comparison of a theoretical estimate of the computational complexity with the measured times for one update step. Measured in multiples of the summed effort for an average elementary fix-point or floating point operation consisting of multiplication, addition and potentially some copying, the feed-forward iteration cost is proportional to $n_{h}^{2}+$ $(2 d+1) n_{h}+d$ with $n_{h}$ the number of hidden neurons and $d$ the dimension of the input and output. This can be expressed in terms of $n_{w}=(2 d+2) n_{h}+d-1$, the number of weights, leading to $n_{h}=\left(n_{w}-d+1\right) /(2 d+2)$. The effort for the derivative calculation is proportional to $\left(n_{h}^{2}+n_{h}+d n_{h}+d\right) n_{w}$ and the effort for the extended Kalman filter updates is proportional to $d^{2}+2 d^{3}+d n_{w}$ $+n_{w}+3 d^{2} n_{w}+5 d n_{w}^{2}+2 n_{w}^{2}$.

Although the complexity of the matrix inversions in Equation (4) scales as $d^{3}$, in practice, this is not the dominant contribution as $n_{w} \gg d$ can be assumed. The dominant contribution for the full online learning step comes from the term $n_{h}^{2} n_{w}=n_{w}^{3} /(2 d+2)^{2}$ of the gradient calculation of the Kalman filter update. For the pure feed-forward step the leading term is $n_{h}^{2}=n_{w}^{2} /(2 d+2)^{2}$, i.e. a factor $n_{w}$ smaller than for the full online learning step.
These estimates are compared with the measured processing times in Figure 34. Here, the processing times are plotted against the estimated effort for $d=1$ and varying $n_{w}$. The linear relationships and the very similar proportionality coefficients confirm the theoretical estimates. As a by-product we get a typical processing time per elementary optimized floating-point operation of about $0.063 \mathrm{~ms}$.

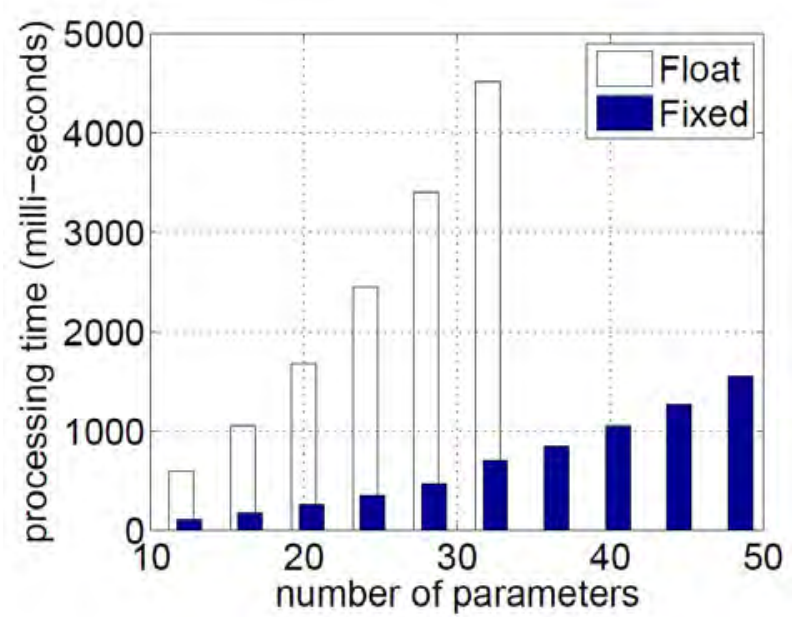

(a) Processing time

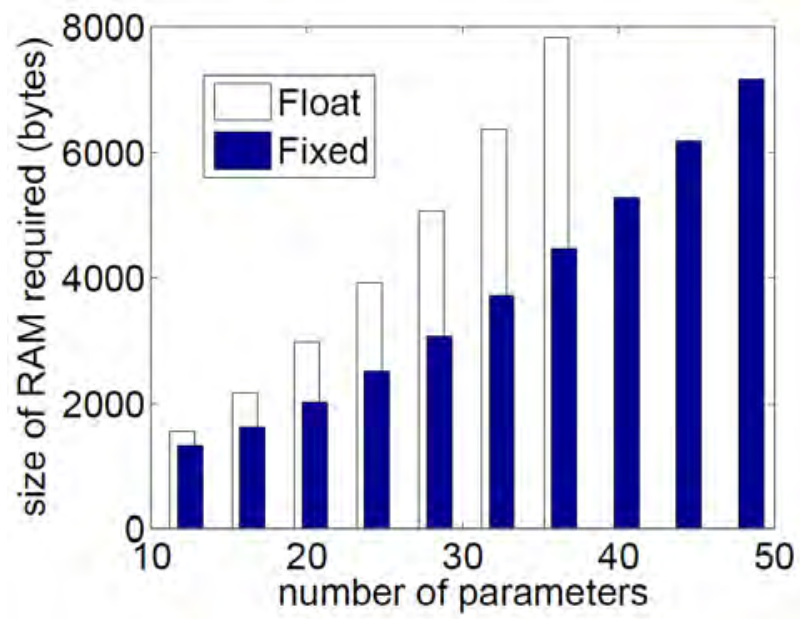

(b) RAM requirement

Figure 32. Comparisons of processing time and RAM Requirement between floating-point models and fixed-point models that installed in TelosB sensor motes with different amounts parameter in model 


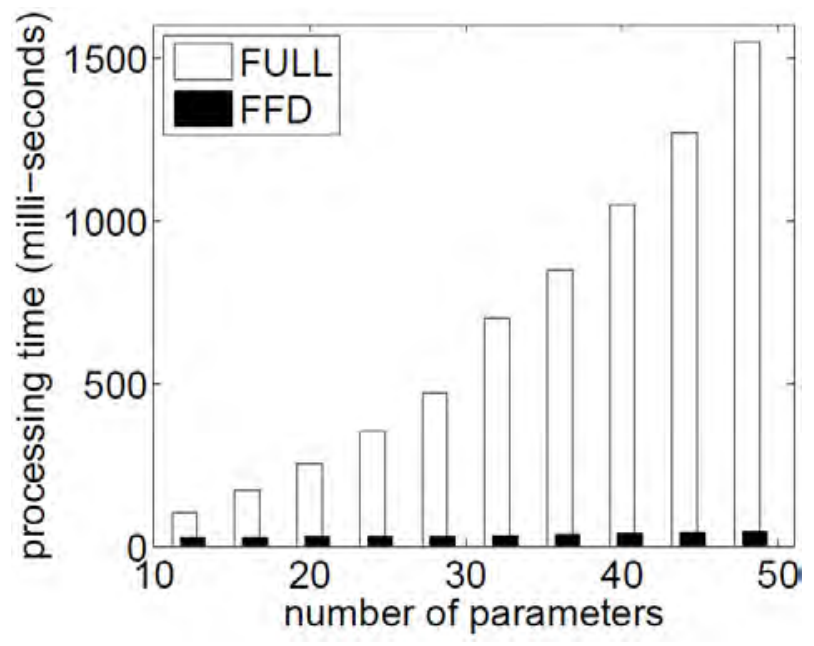

Figure 33. Comparison of processing time between models with feed-forward functionality only and with full functionality (including feed-forwarding, gradient calculation, extended Kalman filter learning)

\section{Conclusions}

Spiral RNN have shown excellent prediction performance and stability during online learning making them a good candidate for application in autonomous systems. A first example are wireless sensor networks where SpiralRNN can be used to reduce duty cycles and thus energy consumption leading to an increased battery lifetime.

Another interesting and not so rare use case is conditional prediction. Here, different temporal pattern are accompanied by an additional constant signal which can be derived from an initial trigger signal. This approach allows training different pattern reliably provided a trigger signal is available. Such trigger signals can also be used to suspend online training, e.g. in periods with just noise on the input.

We have analyzed the storage capacity of SpiralRNN in conditional prediction tasks. It turned out that the number of successfully trained temporal pattern is proportional to the number of hidden neurons. This result is qualitatively similar to the memory capacity of Hopfield networks [15]. As expected, the storage capacity for longer pattern is smaller than the one for shorter pattern. Further investigations might clarify whether there is a typical storage capacity per hidden node and pattern length.

Finally, we have reported about an embedded system implementation of SpiralRNN on a typical wireless sensor platform. A significant reduction of the runtime has been achieved by applying fix-point arithmetics. This allows developing solutions based on online learning of dynamics with reasonably complex models. A typical example is duty cycle adaptation in wireless sensor networks.

\section{Appendix}

The online learning rule can be derived from a Bayesian approach for the probability density $f_{\mathbf{w}_{t} \mid \hat{\mathrm{x}}_{t}}$ of the weights $w$ given all previous data $\hat{\mathrm{X}}_{t}=\left\{\hat{\mathbf{x}}_{t}, \hat{\mathbf{x}}_{t-1}, \ldots, \hat{\mathbf{x}}_{0}\right\}$.

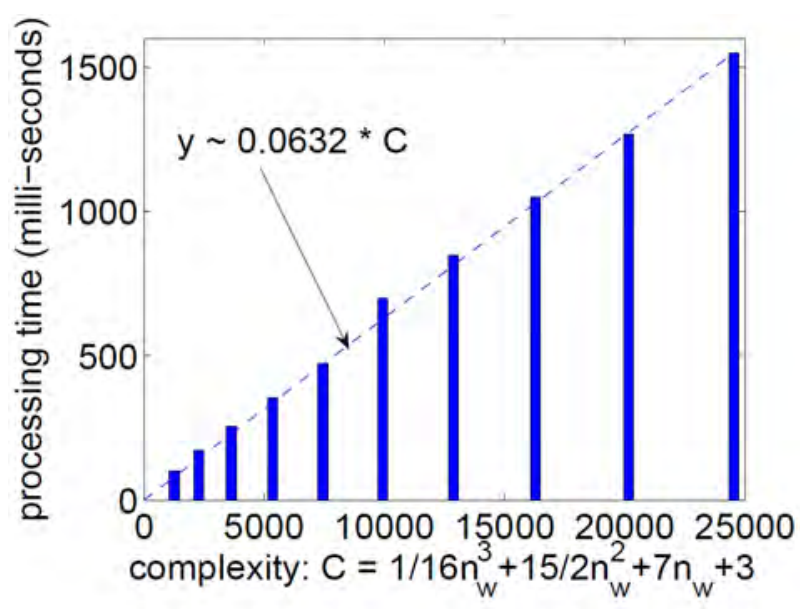

(a) Feedforward

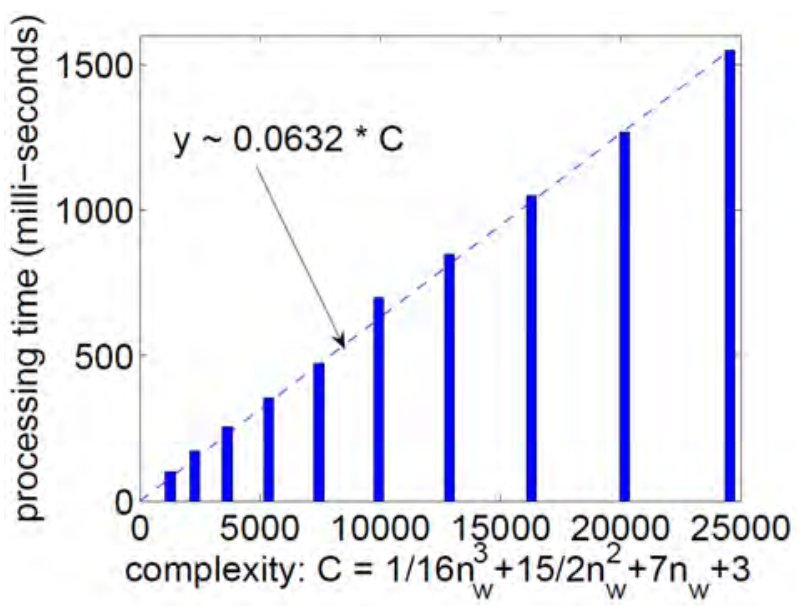

(b) Full online learning step

Figure 34. Processing time as a function of the theoretical estimate of computational complexity for fixed-point implementation: feedforward (top) and full online learning step (bottom). The linear behavior and the almost identical proportionality factors of $0.063 \mathrm{~ms} /$ operation indicate the validity of the theoretical estimate 
We start with a generic dynamic system:

$$
\begin{gathered}
\mathbf{s}_{t}=\mathrm{H}\left(\mathbf{s}_{t-1}, \mathbf{w}_{t}, \hat{\mathbf{x}}_{t-1}\right) \\
\hat{\mathbf{x}}_{t}=\mathrm{G}\left(\mathbf{s}_{t}, \mathbf{w}_{t}, \hat{\mathbf{x}}_{t-1}\right)+v_{t} \\
\mathbf{w}_{t}=\mathbf{w}_{t-1}+\mu_{t}
\end{gathered}
$$

Equation (22) describes the dynamic evolution of the system by a (hidden) state vector $\mathbf{s}_{t}$ at time $t$. The value of $\mathbf{s}_{t}$ depends in some, usually nonlinear way on the previous hidden state vector $\mathbf{s}_{t-1}$, on the previous observation vector $\hat{\mathbf{x}}_{t-1}$ and on the model parameters $\mathbf{w}_{t}$. Equation (23) describes the measurement process: $\hat{\mathbf{x}}_{t}$ is the vector of observations (sensor data) at time $t, \mathrm{G}(\ldots)$ is the model estimation of these observations based on $\mathbf{s}_{t}, \mathbf{w}_{t}$ and $\hat{\mathbf{x}}_{t-1}$. The variable $v_{t}$ is the measurement noise vector, assumed to be normally distributed with a probability distribution function (p.d.f.) $f_{v_{t}}=\mathrm{N}\left(v_{t} \mid 0, R_{t}\right)$. Finally, Equation (24) describes the evolution of the model parameters: that the dynamics of the environment within a reasonable time window are assumed to be stationary, i.e. the model parameters are static, up to additive random fluctuations $\mu_{t}$; these are assumed to be normally distributed with zero mean, covariance matrix $Q_{t}$ and thus with a corresponding p.d.f. $f_{\mu_{t}}=\mathrm{N}\left(\mu_{t} \mid 0, Q_{t}\right)$.

Using the Bayes rule, the chain rule and the Chapman-Kolmogorov Equation (16), the conditional p.d.f. $f_{\mathbf{w}_{t} \mid \hat{\mathrm{X}}_{t}}$ for the model parameters at time $t$ given the current and all previous observations $\hat{X}_{t}=\left\{\hat{\mathbf{x}}_{t}, \hat{\mathbf{x}}_{t-1}, \ldots, \hat{\mathbf{x}}_{0}\right\}$ is given by following equations:

$$
\begin{aligned}
f_{\mathbf{w}_{t} \mid \hat{\mathrm{x}}_{t}} & =f_{\mathbf{w}_{t} \mid \hat{\mathbf{x}}_{t}, \hat{\mathrm{x}}_{t-1}} \\
& =\frac{f_{\hat{\mathbf{x}}_{t} \mid \mathbf{w}_{t}, \hat{\mathrm{x}}_{t-1}} f_{\mathbf{w}_{t} \mid \hat{\mathrm{X}}_{t-1}}}{f_{\hat{\mathbf{x}}_{t} \mid \hat{\mathrm{x}}_{t-1}}} \\
f_{\hat{\mathbf{x}}_{t} \mid \mathbf{w}_{t}, \hat{\mathrm{x}}_{t-1}} & =\int f_{\hat{\mathbf{x}}_{t} \mid \mathbf{s}_{t} \mathbf{w}_{t}, \hat{\mathrm{x}}_{t-1}} f_{\mathbf{s}_{t} \mid \mathbf{w}_{t}, \hat{\mathrm{x}}_{t-1}} d \mathbf{s}_{t} \\
f_{\mathbf{w}_{t} \mid \hat{\mathrm{x}}_{t-1}} & =\int f_{\mathbf{w}_{t} \mid \mathbf{w}_{t-1}, \hat{\mathrm{x}}_{t-1}} f_{\mathbf{w}_{t-1} \mid \hat{\mathrm{x}}_{t-1}} d \mathbf{w}_{t-1}
\end{aligned}
$$

Equation (25) is the famous Bayes rule, Equation (26) introduces the hidden-state vector $\mathbf{s}_{t}$ into the "evi- dence" p.d.f. and Equation (27) is the "prior" p.d.f. for the model parameters $\mathbf{w}_{t}$. The last equation introduces the conditional p.d.f. $f_{\mathbf{w}_{t-1} \mid \hat{\mathrm{x}}_{t-1}}$ which allows to interpret Equation (25) to Equation (27) as iterative update equations for $f_{\mathrm{w}_{t} \mid \hat{\mathrm{x}}_{t}}$.

Before the on-line training rules from these update equations are derived, one has to introduce some assumptions and approximations for the specification of each p.d.f.:

$\left(\mathbf{S}_{a}\right) f_{\mathbf{w}_{t-1} \mid \hat{\mathrm{x}}_{t-1}} \approx \mathrm{N}\left(\mathbf{w}_{t-1} \mid \overline{\mathbf{w}}_{t-1}, P_{t-1}\right)$ is assumed to be normally distributed with mean $\overline{\mathbf{w}}_{t-1}$ and covariance matrix $P_{t-1}$. In general this p.d.f. will be multi-modal; however, if the environment doesn't change its dynamics too fast and if the model is powerful enough, then the model parameters should become static and this assumption is justified.

$\left(\mathbf{S}_{b}\right) \quad f_{\mathbf{w}_{t} \mid \mathbf{w}_{t-1}, \hat{\mathrm{x}}_{t-1}}=\mathrm{N}\left(\mathbf{w}_{t} \mid \mathbf{w}_{t-1}, Q_{t}\right)$ according to the assumption made in Equation (24).

(S $\left.\mathbf{S}_{c}\right) f_{\mathbf{s}_{t} \mid \mathbf{w}_{t}, \hat{\mathbf{x}}_{t-1}}=\delta\left(\mathbf{s}_{t}-\mathrm{H}\left(\mathrm{H}\left(\ldots, \mathbf{w}_{t}, \hat{\mathbf{x}}_{t-2}\right), \mathbf{w}_{t}, \hat{\mathbf{x}}_{t-1}\right)\right)$ with the dots indicating an iteration of the function $\mathrm{H}$ () which further represents the previous hidden states $\mathbf{s}_{t-\tau}(\tau=1,2, \ldots)$ according to equation (22). The Dirac delta-function $\delta$ () corresponds to a normal distribution with infinitesimally small covariance matrix and reflects the assumption that any uncertainty comes from the measurements and from (random) changes of model parameters.

$\left(\mathbf{S}_{d}\right) f_{\hat{\mathbf{x}}_{t} \mid \mathbf{s}_{t} \mathbf{w}_{t}, \hat{\mathbf{x}}_{t-1}}$ in Equation (26) is normal distributed with mean value $\mathrm{G}\left(\mathbf{s}_{t}, \mathbf{w}_{t}, \hat{\mathbf{x}}_{t-1}\right)$ and covariance matrix $R_{t}$ according to Equation (23), i.e.

$$
f_{\hat{\mathbf{x}}_{t} \mid \mathbf{s}_{t} \mathbf{w}_{t}, \hat{\mathrm{x}}_{t-1}}=\mathrm{N}\left(\hat{\mathbf{x}}_{t} \mid \mathrm{G}\left(\mathbf{s}_{t}, \mathbf{w}_{t}, \hat{\mathbf{x}}_{t-1}\right), R_{t}\right) .
$$

With the specifications $\left(\mathbf{S}_{a}\right)$ and $\left(\mathbf{S}_{b}\right)$, Equation (27) is a convolution of normal distributions and thus $f_{\mathrm{w}_{t} \mid \hat{\mathrm{X}}_{t-1}}$ can be easily calculated:

$$
\begin{aligned}
f_{\mathbf{w}_{t} \mid \hat{\mathrm{X}}_{t-1}} & =\mathrm{N}\left(\mathbf{w}_{t} \mid \overline{\mathbf{w}}_{t-1}, P_{t}^{\dagger}\right) \\
P_{t}^{\dagger} & =P_{t-1}+Q_{t}
\end{aligned}
$$


Given the $\delta$-function like p.d.f. $f_{\mathbf{s}_{t} \mid \mathbf{w}_{t}, \hat{\mathrm{x}}_{t-1}}$ in specification $\left(\mathbf{S}_{c}\right)$ and the p.d.f. of $f_{\hat{\mathbf{x}}_{t} \mid \mathbf{s}_{t} \mathbf{w}_{t}, \hat{\mathbf{x}}_{t-1}}$ in specification $\left(\mathbf{S}_{d}\right)$, one finds:

$$
\begin{array}{r}
f_{\hat{\mathbf{x}}_{t} \mid \mathbf{w}_{t}, \hat{\mathrm{x}}_{t-1}}=\mathrm{N}\left(\hat{\mathbf{x}}_{t} \mid \mathrm{G}\left(\mathbf{s}_{t}, \mathbf{w}_{t}, \hat{\mathbf{x}}_{t-1}\right), R_{t}\right) \\
\mathbf{s}_{t}=\mathrm{H}\left(\mathrm{H}\left(\ldots, \mathbf{w}_{t}, \hat{\mathbf{x}}_{t-2}\right), \mathbf{w}_{t}, \hat{\mathbf{x}}_{t-1}\right)
\end{array}
$$

The same assumption as made in specification $\left(\mathbf{S}_{a}\right)$ for $f_{\mathrm{w}_{t-1} \mid \hat{\mathrm{X}}_{t-1}}$ - namely the slow change of the model parameters $\mathbf{w}_{t}$ when the model has been well trained justifies another approximation: the function $\mathrm{G}(. .$.$) can$ be linearized with respect to their dependence on the model parameters $\mathbf{w}_{t}$. In particular,

$$
g\left(\mathbf{s}_{t}, \mathbf{w}_{t}, \hat{\mathbf{x}}_{t-1}\right) \approx \mathbf{x}_{t}+\Psi\left(\mathbf{w}_{t}-\overline{\mathbf{w}}_{t-1}\right)
$$

where the output vector $\mathbf{x}_{t}$ and the gradient matrix $\Psi$ are respectively defined as:

$$
\mathbf{x}_{t}=\mathrm{G}\left(\mathbf{s}_{t}^{\dagger}, \overline{\mathbf{w}}_{t-1}, \hat{\mathbf{x}}_{t-1}\right) \quad \text { and } \quad \Psi=\frac{d \mathbf{x}_{t}}{d \overline{\mathbf{w}}_{t-1}}
$$

Finally, the definition of $\mathbf{s}_{t}^{\dagger}$ is given here. According to Equation (30) and Equation (31), $\mathbf{s}_{t}^{\dagger}$ should be the hidden state vector calculated with the previous model parameters $\overline{\mathbf{w}}_{t-1}$. However, this requires a recalculation of the whole history whenever the model parameters change and therefore is not a viable solution for on-line learning in distributive sensor application. Instead, the approximation in Equation (33) is applied, which implies that the model takes $\mathbf{s}_{t}^{\dagger}$ as the flawless hidden value.

$$
\mathbf{s}_{t}^{\dagger} \approx \mathrm{H}\left(\mathrm{H}\left(\ldots, \overline{\mathbf{w}}_{t-2}, \hat{\mathbf{x}}_{t-2}\right), \overline{\mathbf{w}}_{t-1}, \hat{\mathbf{x}}_{t-1}\right)
$$

With the approximations in Equation (31), Equation (32) and Equation (33), the calculation of $f_{\hat{\mathbf{x}}_{t} \mid \mathbf{w}_{t}, \hat{x}_{t-1}}$ in Equation (29) is rewritten as:

$$
\begin{aligned}
& f_{\hat{\mathbf{x}}_{t} \mid \mathbf{w}_{t}, \hat{\mathrm{x}}_{t-1}} \approx \\
& \mathrm{N}\left(\hat{\mathbf{x}}_{t} \mid \mathrm{G}\left(\mathbf{s}_{t}^{\dagger}, \overline{\mathbf{w}}_{t-1}, \hat{\mathbf{x}}_{t-1}\right)+\right. \\
& \left.\Psi\left(\mathbf{w}_{t}-\overline{\mathbf{w}}_{t-1}\right), R_{t}\right)
\end{aligned}
$$

Combining Equation (28) and equation (34), the p.d.f. $f_{\mathbf{w}_{t} \mid \hat{\mathrm{X}}_{t}}$ in Equation (25) ends up with a normal distribution in Equation (35). Note that $P_{t}^{\dagger}$ is computed in Equation (28) and $\Psi$ is the gradient matrix.

$$
\begin{gathered}
f_{\mathbf{w}_{t} \hat{\mathrm{X}}_{t}}=N\left(\mathbf{w}_{t} \mid \overline{\mathbf{w}}_{t}, P_{t}\right) \\
\overline{\mathbf{w}}_{t}=\overline{\mathbf{w}}_{t-1}+P_{t} \Psi^{T} R_{t}^{-1}\left(\hat{\mathbf{x}}_{t}-\mathbf{x}_{t}\right) \\
P_{t}=P_{t}^{\dagger}- \\
P_{t}^{\dagger} \Psi^{T}\left(\Psi P_{t}^{\dagger} \Psi^{T}+R_{t}\right)^{-1} \Psi P_{t}^{\dagger}
\end{gathered}
$$

Equation (35) is consistent with specification $\left(\mathbf{S}_{a}\right)$ whilst Equation (36) and Equation (37) constitute the extended Kalman filter (EKF) (for more details see $[16,17,18])$.

\section{REFERENCES}

[1] T. Schott, "Global megatrends and their effects on the production of the future," in Conference Proceedings, 5th International Conference on Industrial Informatics, Vienna, Austria, Vol. 1, pp. 18, July 23-27, 2007, http://www. indin2007.org/keynote_schott.php.

[2] Wikipedia, Ambient intelligence. http://en.wikipedia.org/wiki/Ambient_intelligence.

[3] X. Cai, N. Zhang, G. Venayagamoorthy, and D. C. I. Wunsch, "Time series prediction with recurrent neural networks using a hybrid pso-ea algorithm,” in Proceedings of IEEE International Joint Conference on Neural Networks, N. Zhang, Ed., Vol. 2, pp. 1647-1652, 2004.

[4] H-G. Zimmermann, H-G. Zimmermann, R. Grothmann, A. Schafer, and C. Tietz, "Dynamical consistent recurrent neural networks,” in Proceedings of IEEE International Joint Conference on Neural Networks IJCNN '05, R. Grothmann, Ed., Vol. 3, pp. 1537-1541, 2005.

[5] J. L. Elman, "Finding structure in time," Cognitive Science, Vol. 14, No. 2, pp. 179-211, 1990.

[6] A. Waibel, T. Hanazawa, G. Hinton, and K. Shikano, "Phoneme recognition using time-delay neural networks,” IEEE Transactions on Acoustics, Speech and Signal Processing, Vol. 37, No. 3, pp. 328-339, 1989.

[7] P. Mastorocostas and J. Theocharis, “On stable learning algorithm for block-diagonal recurrent neural networks, part 1: The RENNCOM algorithm,” IEEE International Joint Conference on Neural Networks, Vol. 2, pp. 815 820, 2004.

[8] H. Jäger, “The 'echo state' approach to analyzing and training recurrent neural networks,” German National Research Center for Information Technology, Technical Report GMD 148, 2001.

[9] H. Gao, R. Sollacher, and H-P. Kriegel, "Spiral recurrent neural network for online learning," in Proceedings of the 15th European Symposium on Artificial Neural Networks, 
ESANN'2007, April 25-27, 2007, Bruges, Belgium, M. Verleysen, Ed., pp. 483-488, 2007.

[10] R. J. Williams and D. Zipser, "A learning algorithm for continually running fully recurrent neural networks," Neural Computation, Vol. 1, pp. 270-280, 1989.

[11] R. Sollacher and H. Gao, "Efficient online learning with spiral recurrent neural networks," in Proceedings of World Conference on Computational Intelligence WCCI '08, 2008.

[12] H. Gao and R. Sollacher, "Conditional prediction of time series using spiral recurrent neural network," in Proceedings of the European Symposium on Artificial Neural Networks-Advances in Computational Intelligence and Learning, Bruges, Belgium, April 23-25, 2008.

[13] J. Polastre, R. Szewczyk, and D. Culler, "Telos: Enabling ultra-low power wireless research,” in The Fourth International Conference on Information Processing in Sensor Networks: Special track on Platform Tools and Design Methods for Network Embedded Sensors, pp. 364-369, 2005.
[14] D. Gay, P. Levis, R. von Behren, M. Welsh, E. Brewer, and D. Culler, "The nesC language: A holistic approach to networked embedded systems,” ACM SIGPLAN Notices, Vol. 38, No. 5, pp. 1-11, 2003. http://www.tinyos.net/.

[15] R. J. McEliece, E. C. Posner, E. R. Rodemich, and S. S. Venkatesh, "The capacity of the Hopfield associative memory,” IEEE Transactions on Information Theory, Vol. 33, No. 4, pp. 461-482, 1987.

[16] F. Lewis, "Optimal estimation: With an introduction to stochastic control theory,” A Wiley-Interscience Publication, ISBN: 0-471-83741-5, 1986.

[17] R. Kalman, “A new approach to linear filtering and prediction problems," Transactions of the ASME-Journal of Basic Engineering, Vol. 82, pp. 35-45, 1960.

[18] G. Welch and G. Bishop, “An introduction to the Kalman filter," University of North Carolina at Chapel Hill, Department of Computer Science, Technical Report, pp. 95-041, 2002. 